\title{
A Structural Approach to Graph Transformation Based on Symmetric Petri Nets
}

\author{
Lorenzo Capra* \\ Università degli Studi di Milano \\ Dipartimento di Informatica, Via Celoria 18, 20133 Milan, Italy
}

\begin{abstract}
Graph Transformation Systems (GTS) and Petri Nets (PN) are two central, theoretically sound, formal models for concurrent or distributed systems. A lot of papers have focused on the relationship between GTS and PN. It is generally accepted that PN are instances of GTS due to the lack of ability to adapt or reconfigure their structure. In this paper, which extends a recent one, we reverse this perspective by presenting a formal definition of GTS in terms of Symmetric Nets (SN). SN are a standard High-Level PN formalism featuring a compact syntax which highlights behavioural symmetries of systems. The major strength lies in the possibility of using well-established tools to edit/efficiently analyze (SN-based) graph transformation models. We follow a structural approach, based on a newly implemented symbolic calculus, to validate Graph Transformation Rules (GTR) and formally verify GTS properties.

In the second part of the paper, we supply a semantic characterization of the SN-based graph transformation model, by carrying out a thorough comparison with the classical, algebraic double-pushout (DPO) approach. We constructively and formally show how to translate any DPO rule to a corresponding, elementary or composite, $\mathrm{SN}$ rule. We treat both injective and non-injective DPO rules/rule matches. The comparison shows that the SN approach is, in some sense, a generalization of the DPO.
\end{abstract}

Keywords: Graph Transformation Systems, Double Pushout, Symmetric Nets, Structural analysis Operational semantics.

\section{Introduction and Related Work}

Graph transformation (or rewriting) 1, 2, introduced around fifty years ago [3, has been successfully proposed as a general, flexible, theoretically sound model for concurrent systems with a dynamical structure. Lots of research has focused on these topics. Many foundational papers describe different models of

\footnotetext{
${ }^{*}$ Corresponding author
} 
graph transformation and make a comparison among them, using the category theory as a generalizing and unifying semantics [4, 5. Many others present applications, especially in the area of networking, software engineering [2], and more recently, biology [6], social networks [7, chemistry [8].

The connections between Graph Transformation Systems (GTS) and other formalisms for concurrent systems, in particular, Petri Nets (PN) 9 and Process Algebra [10, 11], have been deeply investigated. GTS have borrowed some well known behavioural equivalence concepts from Process Algebra [12, which are similar in expressivity, but less flexible.

On the other side, PN are a central model for concurrent or distributed systems and represent a reference for any formalism meant to describe such systems, including GTS. The success of PN is due to several reasons, two of which particularly significant: a distributed state notion (corresponding to PN marking) that makes it easy to represent typical aspects of concurrency such as local conflicts, synchronizations, non-determinism, and the availability of a number of tools/techniques supporting the editing/analysis of models.

It is generally accepted that (classical) PN are instances of GTS, due to the lack of ability to adapt/reconfigure their structure. Some PN extensions have been thus equipped with dynamical reconfiguration capabilities (a survey may be found in [13]), which, however, almost always build on concepts typical of Graph Transformation Rules.

Kreowsky was the first to show that GTS are a generalization of some PN classes, in his seminal work 14 based on the double-pushout (DPO) approach. The idea is to represent a marked $\mathrm{PN}$ as a graph with three different types of nodes (for places, transitions, and tokens) and describe the firing of a PN transition through a rule (derivation). Since then, several encodings of PN classes in terms of GTS have appeared, among which Place/Transitions nets, Condition/Event nets, Elementary Net Systems, Consume-Produce-Read nets. Some PN variants with extra features, such as read/reset/inhibitor arcs, have also been encoded. It would be impossible to supply a complete list of these proposals, let us refer to [15] (and included references) for the earliest, and to [16, 17] for more recent ones.

This paper extends [18 and is somewhat related to most of those mentioned above. [18] considers the relationship between GTS and PN from a completely new perspective, by proposing a formalization of Graph Transformation Rules in terms of Symmetric Nets (SN) [19. SN are a subclass of Coloured Petri nets 20, 21] featuring a particular syntax that outlines model symmetries and allows for efficient, both state-space based and structural, model analysis. The idea in [18 is to map a Graph Transformation Rule (GTR) to a SN transition connected to a couple of places whose state (marking) encodes a graph. [18] only deals with directed simple graphs.

Such an approach enjoys several benefits. You can exploit well-established tools for the editing/analysis of SN, like GreatSPN 22. The Reachability Graph of a SN supplies the interleaving semantics of a GTS . The associated quotient graph (called Symbolic Reachability Graph) [23, which directly comes from a symbolic graph encoding, naturally abstracts from graph isomorphism. Above 
all, some recent advances in SN symbolic structural analysis [24, 25, implemented in SNexpression tool [26] (www.di.unito.it/ depierro/SNex), allow you to efficiently validate rules and verify general properties of a GTS, such as conflicts, mutual-exclusion, semi-flows.

The first part of this paper significantly extends and consolidates the formalization of Graph Transformation Rules in terms of SN introduced in 18. We consider a more general class of graphs, edge-labelled multi-graphs, and provide a sound structural characterization of SN rules by using the symbolic calculus implemented in SNexpression. We show the potential of structural analysis through simple but not trivial examples of property verification, including the use of semi-flows. We introduce a new kind of composite rule able to emulate graph transformations in undetermined contexts, which are peculiar to algebraic approaches to graph transformation.

In the second part, we conduct a thorough comparison of the SN based approach with a classical algebraic one, the double-pushout, or DPO [4]. We do not use the original, categorical pushout notions, but we follow an operational, though rigorous, schema. Starting from injective DPO rules, then considering non-injective ones, we show in a constructive, formal way how to translate any DPO rule to a corresponding, elementary or composite, SN rule. The intent is twofold: provide a sound, semantic characterization of the SN approach to graph transformation and, in a way, promote the interoperability among different formalisms/tools, a must in formal methods area. The comparison shows that the SN-based approach to graph transformation can be viewed as a generalization of (base) DPO. The possibility of encoding richer forms of graph transformations, such as Attributed Graph Rewriting, is part of our ongoing

work. Let us finally remark that the use of the native stochastic extension of SN (SSN) would represent a natural encoding for Stochastic GTS.

All the examples presented in the paper are available in GreatSPN format at github.com/lgcapra/GTS-SN, together with a notebook including the commands to issue to the SNexpression shell for checking some of the structural formulae used in the examples.

The outline of the paper follows: Section 2 contains background notions and formally introduces SN; Sections 3 through 6 present the SN-based, structural approach to graph transformation: the encoding and formal validation of rules, the generation of a symbolic state-transition system which abstracts from graph isomorphism, some examples of property verification, the definition of composite rules; Section 7 formalizes the procedure(s) translating DPO rules into corresponding SN rules, starting from injective DPO rules/matches, then considering non-injective matches of a rule, lastly, non-injective rules; Section 8 draws some conclusion and outlines ongoing work.

\section{Background}

This section collects the background notions used in the rest of the paper, except for double-pushout (DPO) rules, introduced in Section 7.1. The reader 
may refer to [9] and [19] for a detailed description of classical, low-level, PNs and SNs, respectively.

\subsection{Multisets, multi-set functions, and their operations}

A multiset (bag) over a domain $D$ is a map $b: D \rightarrow \mathbb{N}$, where $b(d)$ is the multiplicity of $d$ in $b$. The support $\bar{b}$ is $\{d \in D \mid b(d)>0\}: d$ is said an element of $b(d \in b)$ if and only if $d \in \bar{b}$. A bag whose elements all have multiplicity one is said type-set. We may denote a multiset $b$ as a weighted formal sum of its elements, weights representing multiplicities (omitted, if equal to one). With some overloading, a set symbol may also denote the corresponding type-set bag. The null multiset, i.e., the multiset with an empty support, is denoted $\epsilon_{D}$, or just $\epsilon$ if its domain is implicit. $B a g[D]$ denotes the set of all bags over $D$.

Bag operations.. Let $b_{1}, b_{2} \in B a g[D]$. The sum $b_{1}+b_{2}$, the difference $b_{1}-b_{2}$, and the intersection $b_{1} \cap b_{2}$ are bags in $B a g[D]$ defined, for any $d \in D$, as: $b_{1}+b_{2}(d)=b_{1}(d)+b_{2}(d) ; b_{1}-b_{2}(d)=b_{1}(d)-b_{2}(d)$ if $b_{1}(d) \geq b_{2}(d), 0$ otherwise; $b_{1} \cap b_{2}(d)=\min \left(b_{1}(d), b_{2}(d)\right)$. Two bags $b_{1}, b_{2}$ are said disjoint if $b_{1} \cap b_{2}=\epsilon$ $\left(\overline{b_{1}} \cap \overline{b_{2}}=\emptyset\right)$. Associativity holds for,$+ \cap$ (which are treated as n-ary operators), but not for - . Relational operators apply component-wise, e.g., $b_{1}<b_{2}$ if and only if $\forall d \in D, b_{1}(d)<b_{2}(d)$. Similarly for the others.

The scalar product $k \cdot b_{1}, k \in \mathbb{N}$, is the bag $b_{1}^{\prime} \in B a g[D]$, s.t. for any $d \in D$ $b_{1}^{\prime}(d)=k \cdot b_{1}(d)$. The hybrid notation $b+k, k \in \mathbb{N}$, stands for $b+k \cdot \bar{b}$, the bag obtained from $b$ by increasing the elements' multiplicity by $k$ (thus, $\epsilon+k=\epsilon$ ).

Let $b_{i} \in \operatorname{Bag}\left[D_{i}\right], d_{i} \in D_{i}$ : the bag Cartesian product $b_{1} \times b_{2} \times \ldots b_{\mathrm{n}}$ belongs to $B a g\left[D_{1} \times D_{2} \times \ldots D_{\mathrm{n}}\right]$, and is defined as

$$
b_{1} \times b_{2} \times \ldots b_{\mathrm{n}}\left(\left\langle d_{1}, d_{2}, \ldots, d_{\mathrm{n}}\right\rangle\right)=b_{1}\left(d_{1}\right) \cdot b_{2}\left(d_{2}\right) \cdot \ldots b_{\mathrm{n}}\left(d_{\mathrm{n}}\right) .
$$

Bag-functions. Bag-operators naturally extend to bag-functions. Let $f_{1}, f_{2}$ : $D \rightarrow B a g\left[D^{\prime}\right]$, and $\left.o p \in\{+,-, \cap\}\right]$. $f_{1}$ op $f_{2}: D \rightarrow B a g\left[D^{\prime}\right]$ is defined $f_{1}$ op $f_{2}(d)=f_{1}(d)$ op $f_{2}(d), \forall d \in D$. Analogously, $\overline{f_{1}}: D \rightarrow 2^{D^{\prime}}$ is defined $\overline{f_{1}}(d)=\overline{f_{1}(d)}, \forall d \in D$. As for relational operators, $f_{1}<f_{2}$ if and only if $f_{1}(d)<f_{2}(d), \forall d \in D$. And so forth. The symbol $\epsilon_{D, D^{\prime}}$ (just $\epsilon$ if the domains are implicit) denotes the constant null function of arity $D \rightarrow B a g\left[D^{\prime}\right]$. The notions of type-set bag and disjoint bags apply to bag-functions as well, considering function evaluation to all the arguments.

Let $f_{i}: D \rightarrow B a g\left[D_{i}\right]$. The scalar product $k \cdot f_{i}$ is defined as $k \cdot f_{i}(d), \forall d \in D$; the Cartesian product $f_{1} \times f_{2} \times \ldots f_{\mathrm{n}}$ is a map $D \rightarrow B a g\left[D_{1} \times D_{2} \times \ldots D_{\mathrm{n}}\right]$ such that $f_{1} \times f_{2} \times \ldots f_{\mathrm{n}}(d)=f_{1}(d) \times f_{2}(d) \times \ldots f_{\mathrm{n}}(d), \forall d \in D$. The notation $\left\langle f_{1}, f_{2}, \ldots, f_{\mathrm{n}}\right\rangle$ (called function-tuple) will be used in place of $f_{1} \times f_{2} \times \ldots f_{\mathrm{n}}$.

Let $f: D \rightarrow B a g\left[D^{\prime}\right]$ : the transpose $f^{t}: D^{\prime} \rightarrow B a g[D]$ is defined as, $f^{t}(x)(y)=f(y)(x), \forall x \in D^{\prime}, y \in D$. The function linear extension $f^{*}:$ $B a g[D] \rightarrow B a g\left[D^{\prime}\right]$ is $f^{*}(b)=\sum_{x \in b} b(x) \cdot f(x)$, for any $b \in B a g[D]$. The

\footnotetext{
${ }^{1}$ we use, again, symbol overloading
} 
linear extension applies to function composition as well: let $h: A \rightarrow B a g[B]$, $g: B \rightarrow B a g[C]$, then $g \circ h: A \rightarrow B a g[C]$ is defined as $g \circ h(a)=g^{*}(h(a))$. We shall henceforth use the same symbol for a bag-function and its linear extension.

Finally, let $\left\{f_{i}\right\}$ be a family of functions $f_{i}: D \rightarrow B a g\left[D^{\prime}\right]$. The linear combination $F=\sum_{i} \lambda_{i} \cdot f_{i}, \lambda_{i} \in \mathbb{Z}$, is a function $D \rightarrow B a g\left[D^{\prime}\right]$ if and only if $F(d)\left(d^{\prime}\right)=\sum_{i} \lambda_{i} \cdot f_{i}(d)\left(d^{\prime}\right) \geq 0, \forall d \in D, d^{\prime} \in D^{\prime}$.

\subsection{Symmetric Nets}

Symmetric Nets (SN) [19] (iso.org/standard/43538.html) are a highlevel PN standard formalism featuring a particular syntax which highlights behavioural symmetries of systems. SN show an acceptable trade-off between expressivity and analysis capability. Efficient algorithms are available for both state-space based [23] and structural [24, 25] analysis of SN. Many of these are integrated in GreatSPN 22, a graphical editor for SN, whereas the most recent advances on structural analysis are implemented in SNexpression [26] (www.di.unito.it/ depierro/SNex), a kind of computer-algebra system.

SN are a particular flavour of Colored Petri Nets (CPN) 21. Like in any $\mathrm{PN}$ formalism, the underlying structure is a finite, directed bipartite graph, whose nodes are partitioned in $P \cup T, P$ and $T$ being non-empty sets, holding the places and transitions, respectively. The former, drawn as circles, represent state variables, whereas the latter, events causing local state changes.

The SN formalism admits two kinds of transitions: those drawn as white rectangles represent observable (time-consuming, in Stochastic SN) events, whereas those drawn as tiny black bars, called immediate, model non-observable (logical) events. The latter take priority over the former. As in any high-level PN formalism, (colour) domains are associated with SN nodes. Each edge (called arc in $\mathrm{SN}$ ) is annotated by a function that, given an element of the (edge's) transition's domain, yields a bag defined on the place's domain. In the next sections, we formally introduce the $\mathrm{SN}$ color annotations and semantics.

\subsubsection{SN Color Domains}

The color structure of a $\mathrm{SN}$ model is built upon the basic color classes $\mathcal{C}=\left\{C_{i}, i=1 \ldots, \mathrm{n}\right\}$, finite, pair-wise disjoint sets, which may be (circularly) ordered or partitioned into static subclasses $C_{i, j}$. A color domain $\mathcal{D}$ is a Cartesian product of classes : $\mathcal{D}=\prod_{i=1, \ldots, \mathrm{n}} C_{i}^{e_{i}}, e_{i} \in \mathbb{N}$ being the repetitions of class $C_{i}$ in $\mathcal{D}$. The color domain of place $p, \mathcal{D}(p)$, defines the type of tokens (color-tuples) $p$ may hold; the color domain of transition $t, \mathcal{D}(t)$, instead defines the potential firing instances of $t$ : we use typed local variables, $\operatorname{Var}(t)$, to refer to single elements in a color-tuple of $\mathcal{D}(t)$. Formally, transition variables are projections, as explained below.

The partition of color classes into static subclasses is what determines the symmetry level of an SN model : each static subclass gathers (all and only) system entities of a given type equivalently behaving, therefore, indistinguishable.

\footnotetext{
${ }^{2}$ Originally introduced with the name of Well-formed Nets, in their stochastic extension.
} 
We shall use simple conventions. Capital letters, e.g. C, will denote color classes. A capital letter with a subscript, e.g., $\mathrm{C}_{i}$, will denote a subclass of a given class.

The SN models used in the paper build on two unordered color classes: $\mathrm{N}=\left\{v_{i}, i: 1 \ldots|\mathrm{N}|\right\}$ and $\mathrm{L}=\bigcup_{j: 1 \ldots|\mathrm{L}|} \mathrm{L}_{j}$, with $\mathrm{L}_{j}=\left\{l b_{j}\right\}, \forall j$. Color domains take the form: $\mathrm{N}^{e_{1}} \times \mathrm{L}^{e_{2}}$.

A transition variable will be denoted (with a few exceptions) by a single, (possibly) subscripted lower-case letter, e.g. $n_{1}$, which implicitly indicates the variable's type $(\mathrm{N})$. Subscripts distinguish variables of the same class if it repeatedly occurs in a transition's domain. Otherwise, the subscript is optional. As an example, the color domain of transition $t_{r_{1}}$ (Figure 1 (R1)) is $\mathrm{N}^{3} \times \mathrm{L}$. The transition's variables are $\left\{n_{i}\right\}, i: 1 \ldots 3$, and $l$.

SN nodes may have a neutral color domain consisting of a singleton color class X. In particular, if $\operatorname{Var}(t)=\emptyset$ then $\mathcal{D}(t)=\mathrm{X}$.

\subsubsection{Guards and Transition Instances}

A transition $t$ may have a guard, $g(t)$, a boolean expression on $\mathcal{D}(\mathrm{t})$ built of basic predicates, such that $\operatorname{Var}(g(t)) \subseteq \operatorname{Var}(t)$. The only used in this paper are (we refer to a generic class $\mathrm{C}$ ):

- $c_{1}=(\neq) c_{2}$, true when $c_{1}$ and $c_{2}$ are assigned the same/a different color

- $c_{1} \in \mathrm{C}_{j}$, true when the color assigned to $c_{1}$ belongs to subclass $\mathrm{C}_{j}$;

The default guard is the constant true, and is omitted.

A transition instance is denoted $(t, b), b \in \mathcal{D}(t)$. It may be seen as an assignment (binding) of colors to $\operatorname{Var}(t)$. For example, an instance of transition $t_{r_{1}}$ (Figure 1 (R1)) is $b:\left(n_{1}=v_{2}, n_{2}=v_{1}, n_{3}=v_{3}, l=l b_{1}\right)$. We say that $(t, b)$ is valid if and only if $g(t)(b)=$ true. With transition color domain we shall henceforth refer to the restriction of $\mathcal{D}(\mathrm{t})$ to valid instances of $t$.

\subsubsection{Arc Functions}

An arc is said input if going from a place to a transition, output if going in the opposite direction. In SN there is another type of arc, called inhibitor, drawn with an ending small circle on the transition. SN arcs are annotated by families of functions, denoted (for each type) $\mathrm{I}[p, t], \mathrm{O}[p, t], \mathrm{H}[p, t]$, respectively. Formally, an arc-function $F[p, t]: \mathcal{D}(t) \rightarrow B a g[\mathcal{D}(p)]$ is expressed as a linear combination:

$$
F[p, t]=\sum_{k} \lambda_{k} \cdot T_{k}\left[g_{k}\right], \lambda_{k} \in \mathbb{Z},
$$

where $T_{k}$ is a tuple (Cartesian product) $\left\langle f_{1}, \ldots, f_{\mathrm{h}}\right\rangle$ of class-functions, possibly suffixed by a guard on $\mathcal{D}(t)$ : if $g_{k}(b)=$ true, then $T_{k}\left[g_{k}\right](b)=f_{1}(b) \times \ldots f_{\mathrm{h}}(b)$, otherwise, $T_{k}\left[g_{k}\right](b)=\epsilon$. Scalars in (1) must ensure that the linear combination is a bag-function. If $\mathcal{D}(p)=\mathrm{X}$ then (for simplicity) $F[p, t] \in \mathbb{N}$.

A class-C function $f_{i}$ is a type-set map $\mathcal{D}(t) \rightarrow B a g[\mathrm{C}]$, defined in terms of elementary functions $\mathcal{E}_{\mathrm{C}}=\left\{c_{j},++c_{j},--c_{j}, \mathrm{C}_{q}, A l l\right\}$ : 
- $c_{j}$ (variable, or projection) maps a color-tuple $b \in \mathcal{D}(t)$ to the $j^{\text {th }}$ occurrence of color $\mathrm{C}$ in $b$; if $\mathrm{C}$ is ordered, $++c_{j}\left(--c_{j}\right)$ yields the $\bmod _{|\mathrm{C}|}$ successor (predecessor) of the color bound to $c_{j}{ }^{3}$,

- $\mathrm{C}_{q}$ (defined only if $\mathrm{C}$ is partitioned) and All are constant maps to $\mathrm{C}_{q}$ and $\mathrm{C}$, respectively.

Formally, a class-function $f_{i}$ is recursively defined:

$$
f_{i}= \begin{cases}e, e \in \mathcal{E}_{\mathrm{C}} & \text { or } \\ e \pm f_{i}, e \in \mathcal{E}_{\mathrm{C}} & \end{cases}
$$

where in $(2) \pm$ are meant as set-operations. This syntax/semantics is fully in accordance with that of GreatSPN [22] and SNExpression [26] tools, whereas is slightly different (even if equivalent) from that used in legacy papers on SNs, where class-functions are defined in turn as linear combinations. We shall exploit it when handling non-injective graph morphisms.

As an example, consider the function-tuple $\left\langle n_{1}, n_{2}+n_{3}, \mathrm{~L}_{1}\right\rangle: \mathrm{N}^{3} \rightarrow \mathrm{N}^{2} \times \mathrm{L}$, which annotates the I/O arcs of transition $t_{r}$ in Figure 12 . When evaluated on a color-tuple $\left\langle v_{1}, v_{2}, v_{3}\right\rangle$, it yields the type-set bag $1 \cdot\left\langle v_{1}, v_{2}, l b_{1}\right\rangle+1 \cdot\left\langle v_{1}, v_{3}, l b_{1}\right\rangle$, instead, when evaluated on a color-tuple $\left\langle v_{1}, v_{2}, v_{2}\right\rangle$ yields $1 \cdot\left\langle v_{1}, v_{2}, l b_{1}\right\rangle$. Its semantics is thus different from the sum $\left\langle n_{1}, n_{2}, \mathrm{~L}_{1}\right\rangle+\left\langle n_{1}, n_{3}, \mathrm{~L}_{1}\right\rangle$, which, when evaluated on $\left\langle v_{1}, v_{2}, v_{2}\right\rangle$, results in $2 \cdot\left\langle v_{1}, v_{2}, l b_{1}\right\rangle$.

It may be convenient/necessary to rewrite arc functions as weighted sums of pair-wise disjoint terms, according to:

Property 1 Any SN arc-function $F$ can be equivalently expressed as $F^{\prime}$

$$
F^{\prime}=\sum_{i} \lambda_{i} T_{i}\left[g_{i}\right], \lambda_{i} \in \mathbb{N} \text {, where } \forall i, j, i \neq j: T_{i}\left[g_{i}\right] \cap T_{j}\left[g_{j}\right]=\emptyset .
$$

For example: $\quad 2 \cdot\left\langle\right.$ All $\left.-n_{1}, n_{2}\right\rangle\left[n_{1} \neq n_{2}\right]+1 \cdot\left\langle n_{2}, n_{2}\right\rangle: \mathrm{N}^{\mathrm{k}} \rightarrow \mathrm{N}^{2}, \mathrm{k}>1$

$\equiv 2 \cdot\left\langle\right.$ All $\left.-n_{1}-n_{2}, n_{2}\right\rangle\left[n_{1} \neq n_{2}\right]+3 \cdot\left\langle n_{2}, n_{2}\right\rangle\left[n_{1} \neq n_{2}\right]+1 \cdot\left\langle n_{2}, n_{2}\right\rangle\left[n_{1}=n_{2}\right]$.

\subsubsection{Semantics of $S N$}

A marking $\mathbf{m}$ provides a distributed notion of state. Formally, $\mathbf{m}$ is a $P$ indexed vector such that $\mathbf{m}[p] \in \operatorname{Bag}[\mathcal{D}(p)]$. The elements of $\mathbf{m}[p]$ (the marking of place $p$ ) are said tokens. If $p$ is neutral then (for simplicity) $\mathbf{m}[p] \in \mathbb{N}$.

The firing rule sets the SN interleaving semantics. We assume that missing arcs are annotated by null functions. A transition instance $(t, b)$ has concession in marking $\mathbf{m}$ if and only if:

- $\forall p \in P: \mathrm{I}[p, t](b) \leq \mathbf{m}[p]$

- $\forall p \in P \forall x \in \mathrm{H}[p, t](b): \mathrm{H}[p, t](b)(x)>\mathbf{m}[p](x)$

\footnotetext{
${ }^{3}$ in this paper we only use unordered classes
} 
An instance $(t, b)$ having concession in $\mathbf{m}$ is enabled if no higher-priority transition instance has concession in $\mathbf{m}$. In that case, $(t, b)$ may fire, withdrawing the bag $\mathrm{I}[p, t](b)$ from each (input) place $p$ and putting the bag $\mathrm{O}[p, t](b)$ into each (output) place $p$. The reached marking, $\mathbf{m}^{\prime}$, is defined as:

$$
\mathbf{m}^{\prime}[p]=\mathbf{m}[p]-\mathrm{I}[p, t](b)+\mathrm{O}[p, t](b), \forall p \in P
$$

We say that $\mathbf{m}^{\prime}$ is reachable from $\mathbf{m}$ through $(t, b)$, denoted $\mathbf{m}[t, b\rangle \mathbf{m}^{\prime}$.

We call a marking $\mathbf{m}$ vanishing if and only if there is some immediate transition instance which is enabled in $\mathbf{m}$, tangible otherwise. If we set a tangible initial marking $\mathbf{m}_{0}$, and there are no infinite firing sequences of immediate transition instances, we can build the reachability graph (RG) of an SN model.

The RG is an edge-labelled, directed multi-graph $\left(N_{R G}, E_{R G}\right)$ (this structure will be formalized next) whose nodes are tangible markings, defined as follows: $\mathbf{m}_{0} \in N_{R G}$; if $\mathbf{m} \in N_{R G}$, and $\mathbf{m}[t, b\rangle \mathbf{m}_{1}\left[t_{1}, b_{1}\right\rangle \ldots \mathbf{m}_{n}\left[t_{n}, b_{n}\right\rangle \mathbf{m}^{\prime}$, where $\mathbf{m}^{\prime}$ is a tangible marking and $\left\{\left(t_{i}, b_{i}\right), i: 1 \ldots n\right\}, n \geq 0$, is a (possibly empty) sequence of immediate transition instances, then $\mathbf{m}^{\prime} \in N_{R G}$ and $\mathbf{m} \stackrel{t, b}{\longrightarrow} \mathbf{m}^{\prime} \in E_{R G}$.

\subsection{Graphs and Graph Morphisms}

We choose to consider a quite general class of graphs, used in many papers, namely directed, edge-labelled, multi-graphs (parallel edges are allowed). Other possible choices, such as the use of hypergraphs or attributed graphs, will be shortly discussed in section 8 . Let $\Lambda$ be a fixed set of labels.

Graph. A graph $G$ is a 5 -tuple $G=(N, E, s, t, l)$ where

- $N$ is a set of nodes, $E$ is a set of edges, $N \cap E=\emptyset$

- $s: E \rightarrow N$ is the source function; $t: E \rightarrow N$ is the target function

$-l: E \rightarrow \Lambda$ is the labelling function

The components of a graph will be subscripted by the graph's name, if needed. The source and target nodes of an edge $e \in E$ are said incident to $e$. A crucial notion is that of (total) graph morphism. Let $G, H$ be graphs.

Graph Morphism. A graph morphism $\phi: G \rightarrow H$ is a pair of functions $\phi_{N}: N_{G} \rightarrow N_{H}, \phi_{E}: E_{G} \rightarrow E_{H}$, such that $\forall e \in E_{G}:$

$$
s_{H}\left(\phi_{E}(e)\right)=\phi_{N}\left(s_{G}(e)\right), t_{H}\left(\phi_{E}(e)\right)=\phi_{N}\left(t_{G}(e)\right), l_{H}\left(\phi_{E}(e)\right)=l_{G}(e)
$$

A morphism maps the nodes and the edges of a graph to the nodes and the edges of another, by preserving graph structure and edge labels. A morphism $\phi: G \rightarrow H$ is said injective (surjective) if both $\phi_{N}$ and $\phi_{E}$ are. If $\phi$ is both injective and surjective $\phi$ is said an isomorphism, written $G \cong H$. The set of morphisms between multi-graphs $G \rightarrow H$ has an associated, obvious equivalence relation: $\phi_{1}, \phi_{2}$ are equivalent if they coincide, up to the mapping of parallel edges. We implicitly consider the representatives of equivalence classes.

Graph morphisms are base components of graph transformation rules and are used to match the left-hand side of a rule to a host graph. 
Another key concept is that of graph gluing, usually called pushout in the categorical setting. The term gluing has an intuitive explanation: assume that $G$ and $H$ are two graphs with a common interface (i.e., an overlap) $I$, then gluing $G$ and $H$ through $I$, results in a graph, denoted $G+_{I} H$, obtained by "joining" $G$ and $H$ via their overlap $I$. The embedding of $I$ into $G$ and $H$ is formally described by two morphism $\phi_{H}: I \rightarrow H, \phi_{G}: I \rightarrow G$. The intuition above is valid if both morphisms are injective, whereas is partially incorrect if they are not: in such a case, in fact, there may be some merge of graph elements, formally captured by definition below using an equivalence class.

Given a set $A$, and an equivalence relation $\equiv$ on $A$, the set of equivalence classes is denoted $A / \equiv$, whereas the equivalence class of $a \in A$ is denoted $[a]_{\equiv}$. In all subsequent definitions, we assume that node/edge sets are disjoint.

Graph Gluing. Let $I, G, H$, be graphs, and $\phi_{H}: I \rightarrow H, \phi_{G}: I \rightarrow G$ be graph morphism ( $I$ is called interface). Let $\equiv$ be the smallest equivalence relation on $E_{G} \cup N_{G} \cup E_{H} \cup N_{H}$ such that $\forall x \in N_{I} \cup E_{I} \phi_{H}(x)=\phi_{G}(x)$. The gluing of $G, H$, over $I$, (denoted $G+_{I} H$ or $G+_{\phi_{H}, \phi_{G}} H$ ) is a graph $X$ such that

$$
\begin{gathered}
N_{X}=\left(N_{G} \cup N_{H}\right) / \equiv \quad E_{X}=\left(E_{G} \cup E_{H}\right) / \equiv \\
s_{X}\left([e]_{\equiv}\right)=\left\{\begin{array}{l}
{\left[s_{G}(e)\right]_{\equiv} \text { if } e \in E_{G}} \\
{\left[s_{H}(e)\right]_{\equiv} \text { if } e \in E_{H}}
\end{array} \quad t_{X}\left([e]_{\equiv}\right)=\left\{\begin{array}{l}
{\left[t_{G}(e)\right]_{\equiv} \text { if } e \in E_{G}} \\
{\left[t_{H}(e)\right]_{\equiv} \text { if } e \in E_{H}}
\end{array}\right.\right. \\
l_{X}\left([e]_{\equiv}\right)=\left\{\begin{array}{l}
l_{G}(e) \text { if } e \in E_{G} \\
l_{H}(e) \text { if } e \in E_{H}
\end{array}\right.
\end{gathered}
$$

Intuitively, we obtain $G+{ }_{I} H$ by juxtaposing the parts of $G$ and $H$ that do not belong to the images of $\phi_{H}, \phi_{G}$, and by merging the elements of $G$ and $H$ with a common pre-image.

\section{Encoding Graph Transformation Rules in SN}

In this section, we formally introduce the graph transformation model based on SN, focusing on graph encoding and structural conditions featuring welldefined rules. We consider by now elementary rules encoded by single transitions. The benefits of this approach are basically three: the possibility of using a symbolic structural calculus for validating single rules and verifying properties of GTS; the (implicit) abstraction from graph isomorphisms when using a symbolic encoding of graphs; the availability of well-established tools supporting SN, like GreatSpn and SNExpression. We shall use a few examples to illustrate the base concepts.

Even if we refer to directed (edge labelled) multi-graphs, we may easily extend this approach to other classes of graphs, e.g. multipartite graphs. 


\subsection{Graph Encoding as an SN Marking}

The basic idea is to encode a graph through a pair of SN places, Node and Edge, which are then suitably connected to any transition (subnet) representing a rule.

Two basic color classes $\mathrm{N}, \mathrm{L}$ are used: $\mathrm{N}=\left\{v_{i}, i: 1 \ldots|\mathrm{N}|\right\}$ holds node descriptors, and is assumed large enough to cover all possible evolutions of a graph; $\mathrm{L}=\mathrm{L}_{1} \cup \mathrm{L}_{2} \cup \ldots \mathrm{L}_{m}$ holds label descriptors, i.e., $\mathrm{L}_{i}=\left\{l b_{i}\right\}$. Their color domains are: $\mathcal{D}($ Node $)=\mathrm{N}, \mathcal{D}($ Edge $)=\mathrm{N}^{2} \times \mathrm{L}$.

A graph $G=\left(N_{G}, E_{G}, s, t, l\right)$ is encoded as an SN marking, denoted $\mathbf{m}_{G}$. Whenever possible, we use the same symbols for graph nodes, edge labels, and corresponding colors of classes $\mathrm{N}$ and L, respectively. We assume $\left|N_{G}\right| \leq|\mathrm{N}|$

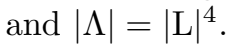

$$
\mathbf{m}_{G}[\text { Node }]=\sum_{v_{i} \in N_{G}} v_{i} \quad \mathbf{m}_{G}[\text { Edge }]=\sum_{e \in E_{G}}\langle s(e), t(e), l(e)\rangle
$$

Observe that $\mathbf{m}_{G}$ [Node] is a type-set bag, whereas the elements of $\mathbf{m}_{G}$ [Edge] may have a multiplicity greater than one (denoting parallel edges).

In general, the class-N colors describing the nodes of graph $G$ in $\mathbf{m}_{G}$ are denoted by an injective map $\operatorname{col}_{G}: N_{G} \rightarrow \mathrm{N}$.

Vice-versa, the following definition sets the conditions under which an SN marking encodes a graph:

Definition 1 (Graph Encoding) Marking $\mathbf{m}$ is a graph-encoding if and only if

- $\mathbf{m}[$ Node] is type-set

$-\left\langle n_{1}+n_{2}\right\rangle \circ \mathbf{m}[$ Edge $] \leq \mathbf{m}[$ Node $]$

The 2nd condition in Definition 1 means that there are no dangling edges, i.e., edges with non-encoded, incident nodes. The graph corresponding to a graphencoding $\mathbf{m}$ is denoted $G_{\mathbf{m}}$ (we skip its formalization, as trivial).

The adopted graph-encoding is not the only possible. A compact alternative might just use place Edge, and the extra subclass $\mathrm{L}_{0}=\left\{l b_{0}\right\}$ : isolated nodes would be represented by color tuples $\left\langle v_{i}, v_{i}, l b_{0}\right\rangle$. This choice, however, would require to check for new (non-)isolated nodes after any edge deletion (insertion).

\subsection{SN Graph Transformation Rules: a Structural Approach}

Graph Transformation Rules (GTR) are formalized as SN subnets including places Node and Edge. We first consider elementary rules consisting of single observable transitions linked to these places (shared among the rules of a GTS), then we introduce and shortly discuss on composite rules, described by more complex subnets.

\footnotetext{
${ }^{4}$ We may also write $N_{G} \subseteq \mathrm{N}, \Lambda \cong L$
} 
An elementary GTR is implemented by a SN transition $t_{r}$, whose color domain is implicitly defined by $\operatorname{Var}\left(t_{r}\right)$ : in general, $\mathcal{D}\left(t_{r}\right)=\mathrm{N}^{e_{1}} \times \mathrm{L}^{e_{2}}, e_{1}>0$.

The idea is simple: the input arc functions I[Node, $\left.t_{r}\right]$, I[Edge, $\left.t_{r}\right]$ (which should not be both null), and the inhibitor arc function $\mathrm{H}\left[\mathrm{Edge}, t_{r}\right]$, when evaluated on an enabled instance of $t_{r}$ in a graph-encoding $\mathbf{m}$, match a subgraph of the encoded graph, which is rewritten according to the SN firing rule: instantaneously, the matched subgraph is removed from the encoded graph and replaced with another subgraph yielded by evaluating the output arc functions on the same instance. The role of inhibitor arc-functions is to set the rule's application context (e.g., a node without predecessors) and ensure general conditions of correctness (e.g., the dangling edge condition).

Figure 1 is a gallery of rule representatives. R1 (when repeatedly applied) builds the (label-preserving) transitive closure of a graph: it requires an initial simple graph to properly work. R2 removes isolated nodes from a graph. R3 derives a Kripke structure from a source graph, by creating an edge-loop with label $l b_{1}$ for nodes without successors. R4 removes parallel edges, if any. R5 deletes a node with only one incident edge-loop: the quite complex inhibitor arc function ensures that there are no dangling edges. Finally, R6 is matched by a couple of edges with source $v_{i}$ and target different from $v_{i}$, with the same label: these two edges are replaced by parallel edges with source $v_{i}$ and target a fresh new node (bound to $n_{4}$ ), with label $l b_{1}$.

\subsection{Elementary Graph Transformation Rules}

A transition $t$ represents a Graph Transformation Rule if any instance of $t$ transforms a graph-encoding into another one, as formalized by:

Definition 2 (Graph Transformation Rule, GTR) A SN transition $t$ is a GTR if and only if, for any graph-encoding $\mathbf{m}, \forall b \in \mathcal{D}(t)$ :

$$
\mathbf{m}[t, b\rangle \mathbf{m}^{\prime} \Rightarrow \mathbf{m}^{\prime} \text { is a graph-encoding. }
$$

Definition 2 refers to any graph/transition instance. Therefore, we need for parametric structural conditions ensuring that an SN transition represents a GTR. By exploiting the SN calculus introduced in [25, 24 and recently implemented in SNexpression tool 26, we can mechanically derive these conditions from the arc-functions surrounding a transition and efficiently manipulate the corresponding symbolic expressions.

SNexpression is a kind of symbolic calculator, which allows checking structural properties (conflict, causal connection, mutual exclusion, semiflows, and many others lower-level) directly on an SN, without any unfolding. The SN calculus solves algebraically any formulae defined in terms of a language, $\mathcal{L}$, and a base set of functional operators (difference, intersection, composition, transpose, and support). The syntax of $\mathcal{L}$ is a small extension of arc-functions' syntax, in which guards defined on functions' co-domain may prefix functions, performing as filters: letting $f: A \rightarrow B a g[B]$, then $[g] f: A \rightarrow B a g[B]$ is such that if 

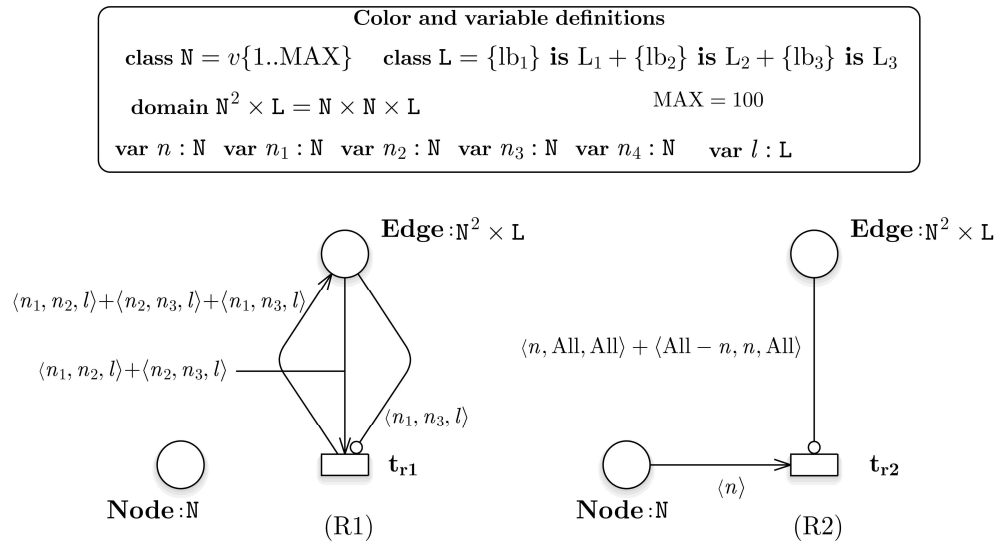

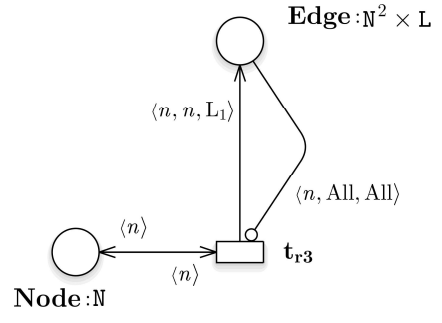

(R3)

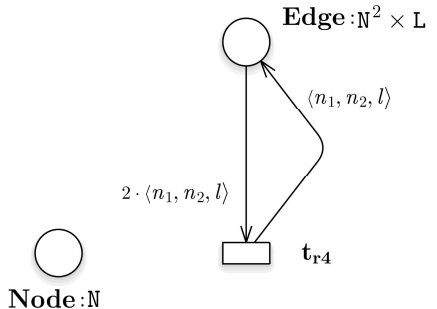

(R4)

Node: $N$

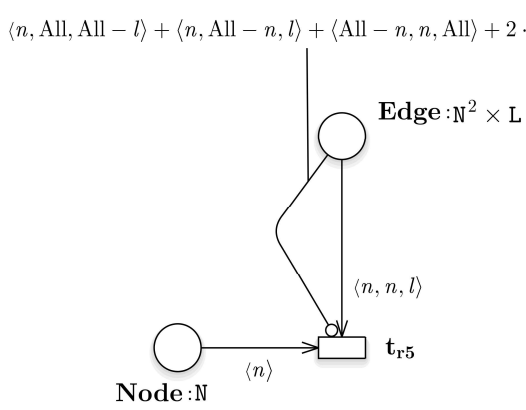

(R5)

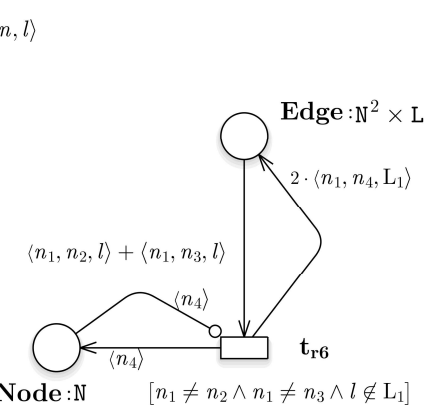

Figure 1: Examples of elementary Graph Transformation Rules 
Table 1: Symbolic expressions used in SN GTR structural definition.

\begin{tabular}{|c|c|}
\hline Symbol & Definition/Description \\
\hline \multicolumn{2}{|r|}{$\mathcal{D}(t) \rightarrow B a g[\mathcal{D}(p)]$} \\
\hline $\mathrm{W}^{+}[p, t]$ & $\begin{array}{l}\mathrm{O}[p, t]-\mathrm{I}[p, t] \\
\text { multi-set of colors put in place } p \text { by an instance of } t\end{array}$ \\
\hline $\mathrm{W}^{-}[p, t]$ & $\begin{array}{l}\mathrm{I}[p, t]-\mathrm{O}[p, t] \\
\text { multi-set of colors removed from } p \text { by an instance of } t\end{array}$ \\
\hline \multicolumn{2}{|r|}{$\mathcal{D}(t) \rightarrow B a g[\mathcal{D}($ Edge $)]$} \\
\hline$F^{*}$ & $\begin{array}{l}\left\langle\mathrm{W}^{-}[\text {Node }, t], A l l, A l l\right\rangle+\left\langle A l l-\mathrm{W}^{-}[\text {Node }, t], \mathrm{W}^{-}[\text {Node }, t], A l l\right\rangle \\
\text { "set" of edges incident to any node removed by an instance } \\
\text { of } t\end{array}$ \\
\hline $\mathrm{W}^{-}[\text {Edge }, t]^{*}$ & $\begin{array}{l}\sum_{i} \lambda_{i} \cdot F_{i} \cap F^{*} \text {, letting } \mathrm{W}^{-}[\text {Edge, } t]=\sum_{i} \lambda_{i} \cdot F_{i} \\
\text { multi-set of edges removed by an instance of } t \text { which are } \\
\text { incident to withdrawn nodes }\end{array}$ \\
\hline
\end{tabular}

$g(x)=$ true then $[g] f(y)(x)=f(y)(x)$, otherwise $[g] f(y)(x)=\epsilon, \forall y \in A, x \in B$. SNexpression works as a rewriting system by reducing any expression $e$, possibly involving the operators mentioned above, to a normal form $e^{\prime} \in \mathcal{L}$, a kind of disjunctive normal form in which the only admitted operators are ' + ' and (at class-function level) ' $\cap$ '. We denote it: $e \rightarrow e^{\prime}$.

SNexpression manages both bag-functions and their supports. In the sequel, we shall use the overloaded operator symbols, '-,,$+ \cap$ ', denoting either bag- or set-operators, depending on their operands. We can syntactically check the equivalence between expressions, thanks to the following property:

$$
e \equiv \epsilon(\emptyset) \Leftrightarrow e \rightarrow \epsilon(\emptyset)
$$

In particular, we shall implicitly use two intuitive equivalences. Let $F, F^{\prime}$ be bag-functions with the same (co-)domains:

$$
F \leq F^{\prime} \Leftrightarrow F-F^{\prime} \equiv \epsilon \quad \bar{F} \subseteq \overline{F^{\prime}} \Leftrightarrow \bar{F}-\overline{F^{\prime}} \equiv \emptyset .
$$

Lemma 1 states quite general conditions for a SN transition $t$ to represent a GTR. The first one is related to the assumption that the marking of place Node is type-set, whereas the other two, a bit more complex, concern dangling-edges. We use some auxiliary expressions, defined and described in Table 1.

Lemma 1 Let $t$ be a SN transition linked to places Node, Edge. If the following conditions hold:

C 1. $\quad \mathrm{H}[$ Node,$t] \leq\langle A l l\rangle \wedge \mathrm{W}^{+}[$Node,$t] \leq \mathrm{H}[$ Node,$t]$

C 2. $\left\langle n_{1}+n_{2}\right\rangle \circ \mathrm{O}[$ Edge,$t]-\left\langle n_{1}+n_{2}\right\rangle \circ \mathrm{I}[$ Edge,$t] \leq \mathrm{O}[$ Node,$t]$

C 3. $\quad \mathrm{H}[$ Edge, $t] \equiv H_{1}+H_{2}, H_{1} \cap H_{2} \equiv \epsilon$

$$
\text { with } H_{1}=\left(F^{*}-\mathrm{W}^{-}[\text {Edge }, t]\right)+\left(\mathrm{W}^{-}[\text {Edge }, t]^{*}+1\right)
$$

then $t$ is a GTR. 
Proof. Let $\mathbf{m}$ be a graph-encoding, $b \in \mathcal{D}(t)$, and $\mathbf{m}[t, b\rangle \mathbf{m}^{\prime}$. The 1 st inequality in Condition 1 ensures that $\mathrm{H}[$ Node, $t](b)$ is a type-set bag: due to the enabling condition, it means that no element of this bag and (for the 2 nd inequality) of the bag added to place Node, which is in turn type-set, must be present in $\mathbf{m}$ [Node]. Consequently, $\mathbf{m}^{\prime}$ [Node] is type-set. Condition 2) avoids the creation of dangling edges due to insertion of edges: $\mathrm{O}[\mathrm{Edge}, t](b)$ is the bag of newly added edges, the minuend consequently is the set of nodes to which these edges are incident. By subtracting the nodes to which existing edges are incident (which are encoded, due to Definition 1) we get the fresh nodes involved in $\mathrm{W}^{+}[$Edge, $t](b)$, enforcing that they are contextually put in place Node. Condition 3) avoids the creation of dangling edges due to node removal. First, observe that $\mathrm{W}^{-}[$Node, $t](b)$, the bag of withdrawn nodes, is actually type-set, otherwise $(t, b)$ would not be enabled in $\mathbf{m}$. The inhibitor arc function ensures that, for every withdrawn node, there is no edge incident to it, but for those contextually removed. The term $t_{1}=\left(F^{*}-\mathrm{W}^{-}[\right.$Edge, $\left.t]\right)(b)$ is (by definition) a type-set symbolic bag representing all and only the edges incident to any removed node, with the exclusion of those which are contextually withdrawn; $t_{2}=\left(\mathrm{W}^{-}[\text {Edge }, t]^{*}+1\right)(b)$ is a multi-set including all and only the withdrawn edges which are incident to any withdrawn node, with their multiplicity increased by one. The two terms above are disjoint by construction. Since the inhibitor arc function $\mathrm{H}[\mathrm{Edge}, t]$ includes both terms, plus (possibly) another one disjoint as well, and $(t, b)$ is enabled, we have: if $x \in t_{1}$, i.e., $x$ is an edge incident to a removed node, which is not contextually removed, then $x$ is not encoded; otherwise, if $x \in t_{2}$, i.e., $x$ is an edge incident to a removed node, and $x$ is contextually removed, then it is encoded in exactly as many instances as those withdrawn (formally, it holds I Edge, $t](b)(x) \leq \mathbf{m}[$ Edge $](b)(x)$ and $\mathrm{H}[$ Edge, $t](b)(x)=\mathrm{W}^{-}[$Edge,$t](b)(x)+1>\mathbf{m}[$ Edge $](b)(x)$, thus, since $\mathrm{W}^{-}[p, t] \leq \mathrm{I}[p, t]$, we get: $\mathrm{I}[$ Edge,$t](b)(x)=\mathbf{m}[$ Edge $\left.](b)(x)\right)$.

Let us point out two relevant aspects of Lemma 1 .

1. No constraint is set on I[Node, $t]$ because Definition 2 only concerns enabled instances. Since we assume that the marking of place Node is type-set, every instance $(t, b)$ such that I[Node, $t](b)$ is not type-set is excluded. Thus, there may be implicit guards: for example, if I[Node, $t]=\left\langle n_{1}\right\rangle+\left\langle n_{2}\right\rangle$, it is implicit $n_{1} \neq n_{2}$, if I $[$ Node, $t]=\left\langle A l l-n_{1}\right\rangle+\left\langle n_{2}\right\rangle$, it is implicit $n_{1}=n_{2}$.

2. Condition 3 ) explicitly shows what $\mathrm{H}[\mathrm{Edge}, t]$ looks like: this makes it possible to mechanically derive it from $\mathrm{W}^{-}[$Node, $t]$ and $\mathrm{W}^{-}[$Edge,$t]$.

We have successfully verified the conditions set by Lemma 1 on all transitions shown in Figure 1, by means of SNexpression. The check, basically a termequivalence test, took (at most) a few dozen ms for each involved calculation.

Structured Labels. In our encoding of graphs edge labels are coherently represented as elementary objects, corresponding to singleton subclasses of L. To enhance the model's expressivity, we might, however, use richer structured labelling of edges, and retain all the theoretical achievements. A label could be 
expressed as a color-tuple of whatever color domain, on which all available SN functions could apply. This goes in the direction of those graph rewriting extensions introduced for modelling reasons, like Attributed Graph Rewriting [27, 28 . In particular, the characterization of SN Graph Transformation Rules provided by Lemma 1 would still work, but for inserting as many occurrences of the All constant function as the label domain's size, at the end of function-tuples. Also, all the outcomes of the next sections would apply with a few adaptations.

\subsection{Graph Transformation System}

An SN Graph Transformation System consists of an initial graph and a set of SN graph transformation rules.

Definition 3 (SN Graph Transformation System) Let $G_{0}$ be a graph, and $\mathcal{R}$ be a set of SN Graph Transformation Rules (Definition 2). The corresponding GTS is obtained by sharing places Node and Edge among $\mathcal{R}$ transitions, and setting $\mathbf{m}_{G_{0}}$ as initial marking.

The GTS state-transition system corresponds to the SN Reachability Graph.

Consider, as an example, the SN in Figure 2 bringing together the elementary rules R1,R3 of Figure 1 1 . Given a graph $G_{0}$ encoded by the initial marling $\mathbf{m}_{G_{0}}$, the SN Reachability Graph describes the sequence of transformations that $G_{0}$ undergoes by applying either Rule 1 or Rule 3. The resulting RG has an absorbing state, i.e. a dead home-state, which corresponds to the transitive closure of $G_{0}$, where, also, nodes without proper predecessors have newly added, incident edge-loops.

Let $\mathbf{m}_{G_{0}}[$ Edge $]=\left\langle v_{1}, v_{2}, l b_{1}\right\rangle+\left\langle v_{1}, v_{3}, l b_{1}\right\rangle+\left\langle v_{4}, v_{1}, l b_{1}\right\rangle$, and $\mathbf{m}_{G_{0}}[$ Node $]=$ $\left\langle v_{1}+v_{2}+v_{3}+v_{4}\right\rangle$ : the corresponding RG (built with the GreatSPN package) holds 16 nodes, including the absorbing one:

$\left\langle v_{1}, v_{2}, l b_{1}\right\rangle+\left\langle v_{1}, v_{3}, l b_{1}\right\rangle+\left\langle v_{4}, v_{1}, l b_{1}\right\rangle+\left\langle v_{2}, v_{2}, l b_{1}\right\rangle+\left\langle v_{3}, v_{3}, l b_{1}\right\rangle+\left\langle v_{4}, v_{2}, l b_{1}\right\rangle+\left\langle v_{4}, v_{3}, l b_{1}\right\rangle$

\section{Graph Isomorphism Abstraction and SN Symbolic Marking}

In this section, we introduce the SN Symbolic Marking notion (with some related concepts) and discuss the isomorphism abstraction it implicitly provides in the graph encoding context.

In the context of GTS, all considerations are valid up to graph isomorphism. In particular, the state-transition system associated with a GTS usually abstracts from isomorphic graphs obtained by applying rules.

The system symmetries implicitly expressed by the syntax of the SN formalism, in the graph encoding context, exactly match graph isomorphism. Let us recall a few basic concepts and refer to 23 for the details.

$\mathrm{SN}$ are equipped with a syntactical state-equivalence notion, called symbolic marking (SM). Two SN markings $\mathbf{m}_{1}, \mathbf{m}_{2}$ belong to the same SM $\widehat{\mathbf{m}}$ if and only if there is a permutation $\sigma$ on color classes (a rotation, on ordered classes), 
preserving static subclasses, such that $\mathbf{m}_{2}=\sigma\left(\mathbf{m}_{1}\right)$ (we write $\mathbf{m}_{1} \equiv \mathbf{m}_{1}$ ). An immediate consequence is the following.

Property $2 G \cong G^{\prime}$ if and only if there exists a permutation $\sigma$ of class-N colors such that $\mathbf{m}_{G^{\prime}}=\sigma\left(\mathbf{m}_{G}\right)$.

Note that the only permutation on $\mathrm{L}$ is the identity.

We might easily extend this intuitive property if edge labels had a richer structure, as discussed later.

At transition instance level, the SN symmetry looks like a strong bisimulation: for each color permutation $\sigma: \mathbf{m}[t, b\rangle \mathbf{m}^{\prime} \Leftrightarrow \sigma(\mathbf{m})[t, \sigma(b)\rangle \sigma(\mathbf{m})^{\prime}$; on the other side, $\mathbf{m}_{1}[t, b\rangle \mathbf{m}_{2} \Rightarrow \forall \mathbf{m}_{2}^{\prime}, \mathbf{m}_{2}^{\prime} \equiv \mathbf{m}_{2} \exists \mathbf{m}_{1}^{\prime}, b^{\prime}, \mathbf{m}_{1}^{\prime} \equiv \mathbf{m}_{1} \wedge b^{\prime} \equiv b: \mathbf{m}_{1}^{\prime}\left[t, b^{\prime}\right\rangle \mathbf{m}_{2}^{\prime}$.

By setting an initial symbolic marking, $\widehat{\mathbf{m}}_{0}$, we can thus automatically generate (with the GreatSPN package) a quotient graph, called Symbolic Reachability Graph (SRG), which retains all the liveness/safety properties of the ordinary RG of a SN. Formally, a symbolic marking (SM) employs dynamic subclasses instead of colours. Dynamic subclasses define (locally) a parametric partition of color (sub-)classes: each dynamic subclass refers to a specific static subclass, or a colour class if that class is not partitioned. The size of a dynamic subclass indicates a set of different colors evenly distributed over the SN places. Consider, for example, the initial symbolic marking below, where symbol $z v_{i}$ denotes a class-N dynamic subclass, whereas static subclasses denote edge labels:

$$
\widehat{\mathbf{m}}_{0}[\text { Edge }]=\left\langle z v_{1}, z v_{2}, \mathrm{~L}_{1}\right\rangle+\left\langle z v_{3}, z v_{1}, \mathrm{~L}_{1}\right\rangle, \widehat{\mathbf{m}}_{0}[\text { Node }]=\left\langle z v_{1}+z v_{2}+z v_{3}\right\rangle
$$

where $\left|z v_{1}\right|=\left|z v_{3}\right|=1,\left|z v_{2}\right|=2$. This symbolic marking represents a number of equivalent SN markings (six if $\mathrm{N}=4$ ) including $\mathbf{m}_{G_{0}}$ defined at the end of the last section. Observe that colors $v_{2}, v_{3}$ are now folded into $z v_{2}$.

The SRG is directly built from $\widehat{\mathbf{m}}_{0}$ through a symbolic firing mechanism. Leaving out technical details, a symbolic instance of transition $t_{r_{1}}$ (Figure 2) is: $\left(n_{1}=z v_{3}, n_{2}=z v_{1}, n_{3}=z v_{2}\right)$. This symbolic instance, which folds two color instances, is enabled in $\widehat{\mathbf{m}}_{0}$; when firing, it leads to the symbolic marking 5

$$
\left\langle z v_{3}, z v_{1}, \mathrm{~L}_{1}\right\rangle+\left\langle z v_{1}, z v_{2}, \mathrm{~L}_{1}\right\rangle+\left\langle z v_{3}, z v_{2}, \mathrm{~L}_{1}\right\rangle,\left|z v_{2}\right|=2
$$

The SRG of the simple example we are considering contains 10 nodes, one of which absorbing, against the 16 nodes of the corresponding RG.

When considering large graphs, the reduction achieved with the SRG in terms of states/edges may be dramatic. In particular, if a GTR adds new nodes to the encoded graph, GTR's symbolic firing instances may fold together a huge number of ordinary ones. The reason is that added nodes correspond to class$\mathrm{N}$ colors bound to fresh transition variables, like $n_{4}$ in Figure 1 (R6).

A canonical representative for SM permits a syntactical comparison between SM. In the context of graph encoding, bringing an SM into its canonical form has

\footnotetext{
${ }^{5}$ we only refer to place Edge, because the marking of Node doesn't change
} 


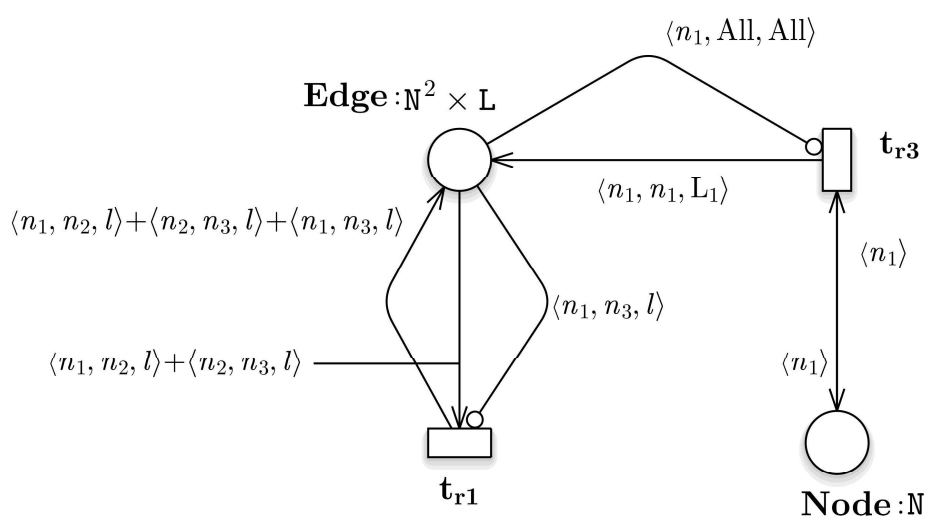

Figure 2: GTS composed of rules R1,R3 of Figure 1

more or less the same complexity as checking graph isomorphism. We believe that the legacy GreatSPN algorithm computing SM canonical representatives (which in addition, for performance analysis purposes, calculates the size of SM by enumerating color permutations) might be significantly improved by using structural analysis outcomes. This is a part of ongoing work.

In the rest of the paper, to keep notations simple, we shall refer to ordinary markings/transition instances.

\section{Exploiting SN Structural Analysis: Application Examples}

In this section, we aim at showing the potential of SN structural analysis in graph transformation field, by means of a simple example of GTS. We introduce/use (symbolic) structural relations and (colored and numerical) semiflows.

Symbolic structural analysis is a promising, effective approach to formal verification of properties, with a lot of positive effects also on traditional methods based on state-space exploration and model-checking.

In Section 3.3, we have set conditions on arc functions characterizing SN Graph Transformation Rules. These conditions, which involve base functional operators on bag-expressions, can be automatically and efficiently checked with the SNexpression tool.

The SN structural calculus allows you not only to validate GTRs, but also to check general properties of a GTS, e.g., figure out which rules are in conflict (as a consequence, may concurrently apply), mutually exclusive, causally connected, and so forth. True concurrent or partial-order approaches have been widely treated in graph rewriting and PN literature: we are not interested in a theoretical discussion about these specific topics, rather we aim at showing the potential of SN structural analysis in these and related research areas. 


\subsection{Symbolic Structural Relations}

A (symbolic) structural relation between SN nodes $e, e^{\prime}$, defined as $\mathcal{R}\left(e, e^{\prime}\right)$ : $\mathcal{D}\left(e^{\prime}\right) \rightarrow 2^{\mathcal{D}(e)}$, maps any instance $b^{\prime}$ of $e^{\prime}$ to the set of instances of $e$ related with $b^{\prime}$. Formally, structural relations are expressions involving arc functions and a base set of functional operators: transpose, sum, intersection, difference, support, and composition.

Two base relations, $\mathrm{Rb}[t, p], \mathrm{Ab}[t, p]$, and others higher-level, are listed in Table 2 with their formulae. $\mathrm{Rb}[t, p]$ (Removed by), given an element $c$ of the color domain of place $p$, results in the set of instances of $t$ that withdraw $c$ from $p ; \mathrm{Ab}[t, p]$ (Added by) is dual, given a color $c$ results in the set of instances of $t$ that put $c$ in $p$. An intuitive explanation of the others follows.

(Asymmetric) Structural Conflict: two transition instances $(t, b)$ and $\left(t^{\prime}, b^{\prime}\right)$ are in a conflict relation in marking $\mathbf{m}$ if the firing of the former disables the latter. The structural conflict relation $(S C)$ sets the necessary conditions for a conflict: $S C\left(t, t^{\prime}\right)$ maps an instance $b^{\prime}$ of $t^{\prime}$ to the set of (all and only) the colour instances $b$ of $t$ that may disable $\left(t^{\prime}, b^{\prime}\right)$. A conflict arises because $(t, b)$ withdraws tokens from an input place of $t^{\prime}$ or it adds tokens to an inhibitor place of $t^{\prime}$. The SC formula is the summation over all local conflicts possibly caused by the situations described above. Different instances of the same transition may be conflicting: the corresponding, one-argument SC formula, therefore, uses the identity function as a final subtrahend.

Structural Causal Connection: two transition instances $(t, b)$ and $\left(t^{\prime}, b^{\prime}\right)$ are in causal connection in a marking $\mathbf{m}$ if the firing of the former causes the enabling of the latter. The structural causal connection $(S C C)$ relation sets the necessary conditions for causal connection: $S C C\left(t, t^{\prime}\right)$ maps an instance $b^{\prime}$ of $t^{\prime}$ to the set of the instances $b$ of $t$ that may cause the enabling of $\left(t^{\prime}, b^{\prime}\right)$. This situation occurs if $(t, b)$ adds tokens to an input place of $t^{\prime}$ or it withdraws tokens from an inhibitor place of $t^{\prime}$.

Structural Mutual Exclusion: two instances $(t, b)$ and $\left(t^{\prime}, b^{\prime}\right)$ are in structural mutual exclusion $(S M E)$ if whenever one is enabled the other is not, and viceversa. This case occurs when there is a place $p$ which is both an input place for $t$ and an inhibitor place for $t^{\prime}$, and the number of tokens of a certain color required for the enabling of $t$ is at least equal to the upper-bound enforced by the inhibitor arc-function for tokens of that color. The symmetric relation $S M E\left(t, t^{\prime}\right)$ maps an instance $b^{\prime}$ of $t^{\prime}$ to the set of instances of $t$ that cannot be enabled when $\left(t^{\prime}, b^{\prime}\right)$ is. The $S M E$ formula given in Table 2 works if all involved input/inhibitor arc-functions are type-set, as in most of SN models presented in this paper. Note that the formula is slightly different if $t=t^{\prime}$. [25] treats the general case of SME.

Applications. Assume that we want to determine which rules (described by SN) of a GTS may simultaneously apply. By using the SN structural calculus, we can give a precise answer. First of all, we should partition the whole set of GTS rules' instances into (symbolic) conflict sets, each representing all the instances which may conflict, directly or indirectly. And even though, in general, this 
Table 2: Symbolic structural relations in SN

\begin{tabular}{|c|c|c|c|}
\hline $\mathrm{Ab}[t, p]$ & $=$ & ${\overline{\mathrm{W}^{+}[p, t]}}^{t}$ & \\
\hline $\operatorname{Rb}[t, p]$ & $=$ & $\overline{\mathrm{W}}^{-}[p, t]{ }^{t}$ & \\
\hline$S C\left(t, t^{\prime}\right)$ & $=$ & $\sum_{p} \mathrm{Rb}[t, p] \circ \overline{\mathrm{I}\left[t^{\prime}, p\right]}+\mathrm{Ab}[t, p] \circ \overline{\mathrm{H}\left[t^{\prime}, p\right]}$ & $t \neq t^{\prime}$ \\
\hline$S C(t)$ & $=$ & $\left(\sum_{p}^{P} \mathrm{Rb}[t, p] \circ \overline{\mathrm{I}[t, p]}+\mathrm{Ab}[t, p] \circ \overline{\mathrm{H}[t, p]}\right)-I d e_{\mathcal{D}(t)}$ & \\
\hline$S C C\left(t, t^{\prime}\right)$ & $=$ & $\sum_{p} \mathrm{Ab}[t, p] \circ \overline{\mathrm{I}\left[t^{\prime}, p\right]}+\mathrm{Rb}[t, p] \circ \overline{\mathrm{H}\left[t^{\prime}, p\right]}$ & \\
\hline$S M E\left(t, t^{\prime}\right)$ & $=$ & $\sum_{p} \overline{\mathrm{I}[t, p]}^{t} \circ \overline{\mathrm{H}\left[t^{\prime}, p\right]}+\overline{\mathrm{H}[t, p]}^{t} \circ \overline{\mathrm{I}\left[t^{\prime}, p\right]}$ & $t \neq t^{\prime}$ \\
\hline$S M E(t)$ & $=$ & $\left(\sum_{p} \overline{\mathrm{I}}[t, p]^{t} \circ \overline{\mathrm{H}[t, p]}\right)-I d e_{\mathcal{D}(t)}$ & \\
\hline
\end{tabular}

requires calculating the symmetric and transitive closure of $S C$ relation 25], in the following examples we can use a smarter technique.

To illustrate the concept, let us consider the GTS in Figure 2, The two rules are potentially in conflict due to place Edge, which is (reciprocally) an output place for one and an inhibitor place for the other. There are no potential conflicts due to shared input places since the corresponding expressions of $\operatorname{Rb}[t, p]$ turn out to be null (by the way, a composition involving null operand results in turn null). As for added by, we get the following non-null expressions: (in the rest of the section, function supports are implicitly used): $\mathcal{D}\left(t_{r_{1}}\right)=\mathrm{N}^{3} \times \mathrm{L}, \mathcal{D}\left(t_{r_{3}}\right)=\mathrm{N}$

$$
\mathrm{Ab}\left[t_{r_{1}}, \text { Edge }\right]=\left\langle n_{1}, A l l, n_{2}, l\right\rangle \quad \mathrm{Ab}\left[t_{r_{3}}, \text { Edge }\right]=\left\langle n_{1}\right\rangle\left[n_{1}=n_{2} \wedge l \in \mathrm{L}_{1}\right]
$$

The expression on the left says that a color tuple $\left\langle c_{1}, c_{2}, l b_{i}\right\rangle$ is put in place Edge by any instance $\left\langle c_{1}, *, c_{2}, l b_{i}\right\rangle$ of $t_{r_{1}}$. The other expression instead says that a color tuple $\left\langle c_{1}, c_{1}, l b_{1}\right\rangle$ is put in place Edge by the instance $\left\langle c_{1}\right\rangle$ of $t_{r_{3}}$.

According to the formula in Table 2 and the tuple-transpose algorithm [24, we obtain, after some rewriting:

$$
\begin{array}{ll}
S C\left(t_{r_{1}}, t_{r_{3}}\right)=\left\langle n_{1}, A l l, n_{2}, l\right\rangle \circ\left\langle n_{1}, A l l, A l l\right\rangle & \equiv\left\langle n_{1}, A l l, A l l, A l l\right\rangle \\
S C\left(t_{r_{3}}, t_{r_{1}}\right)=\left\langle n_{1}\right\rangle\left[n_{1}=n_{2} \wedge l \in L_{1}\right] \circ\left\langle n_{1}, n_{3}, l\right\rangle & \equiv\left\langle n_{1}\right\rangle\left[n_{1}=n_{3} \wedge l \in \mathrm{L}_{1}\right]
\end{array}
$$

Once again, we can supply an intuitive explanation: $S C\left(t_{r_{1}}, t_{r_{3}}\right)$ indicates that an instance $\left\langle c_{1}\right\rangle$ of $t_{r_{3}}$ may be in conflict with any instance $\left\langle c_{1}, *, *, *\right\rangle$ of $t_{r_{1}}$, $S C\left(t_{r_{3}}, t_{r_{1}}\right)$ instead indicates that an instance $\left\langle c_{1}, *, c_{1}, l b_{1}\right\rangle$ of $t_{r_{1}}$ is in conflict with the instance $\left\langle c_{1}\right\rangle$ of $t_{r_{3}}$.

The $S C$ relation points out potential conflicts. We can enhance the previous outcome by computing $S M E$ : Edge is both an input and an inhibitor place for $t_{r_{1}}$, and inhibitor for $t_{r_{3}}$. According to the formula in Table 2, we obtain:

$$
\begin{aligned}
S M E\left(t_{r_{1}}, t_{r_{3}}\right) & =\left(\left\langle n_{1}, n_{2}, A l l, l\right\rangle+\left\langle A l l, n_{1}, n_{2}, l\right\rangle\right) \circ\left\langle n_{1}, A l l, A l l\right\rangle \\
& \equiv\left\langle n_{1}, A l l-n_{1}, A l l, A l l\right\rangle+\left\langle A l l, n_{1}, A l l, A l l\right\rangle
\end{aligned}
$$

$S M E\left(t_{r_{3}}, t_{r_{1}}\right)$ turns out to be $\left\langle n_{1}\right\rangle+\left\langle n_{2}\right\rangle$, i.e., $S M E\left(t_{r_{3}}, t_{r_{1}}\right) \equiv S M E\left(t_{r_{1}}, t_{r_{3}}\right)^{t}$, because $S M E$ is symmetric. But what matters is that:

$$
S C\left(t_{r_{1}}, t_{r_{3}}\right) \subseteq S M E\left(t_{r_{1}}, t_{r_{3}}\right) \wedge S C\left(t_{r_{3}}, t_{r_{1}}\right) \subseteq S M E\left(t_{r_{3}}, t_{r_{1}}\right)
$$


That is, any conflicting instances of $t_{r_{1}}$ and $t_{r_{3}}$ are mutually exclusive. In other words, these two rules are potentially concurrent.

We can also extend this result to instances of the same GTR. According to the formula in Table 2 , we get $\left(I d e_{\mathcal{D}\left(t_{r_{1}}\right)}=\left\langle n_{1}, n_{2}, n_{3}, l\right\rangle\right)$ :

$$
\begin{aligned}
S C\left(t_{r_{1}}\right)= & \left\langle n_{1}, A l l-n_{1}, n_{3}, l\right\rangle\left[n_{1}=n_{2} \wedge n_{1} \neq n_{3}\right]+\left\langle n_{2}, A l l, n_{3}, l\right\rangle\left[n_{1} \neq n_{2}\right]+ \\
& \left\langle n_{1}, A l l, n_{1}, l\right\rangle\left[n_{1}=n_{2} \wedge n_{1} \neq n_{3}\right]+\left\langle n_{1}, n_{2}, n_{2}, l\right\rangle\left[n_{1} \neq n_{2} \wedge n_{2} \neq n_{3}\right]+ \\
& \left\langle n_{1}, A l l-n_{1}, n_{1}, l\right\rangle\left[n_{1}=n_{2} \wedge n_{1}=n_{3}\right]+\left\langle n_{1}, A l l-n_{2}, n_{2}, l\right\rangle\left[n_{1} \neq n_{2}\right]
\end{aligned}
$$

The expression for mutually exclusive instances of GTR $t_{r_{1}}$ is:

$$
\begin{aligned}
S M E\left(t_{r_{1}}\right)= & \left\langle n_{1}, A l l-n_{1}, n_{3}, l\right\rangle\left[n_{1}=n_{2} \wedge n_{1} \neq n_{3}\right]+\left\langle n_{2}, A l l, n_{3}, l\right\rangle\left[n_{1} \neq n_{2}\right]+ \\
& \left\langle n_{1}, A l l, n_{1}, l\right\rangle\left[n_{1}=n_{2} \wedge n_{1} \neq n_{3}\right]+\left\langle n_{1}, n_{2}, n_{2}, l\right\rangle\left[n_{1} \neq n_{2} \wedge n_{2} \neq n_{3}\right]+ \\
& \left\langle n_{1}, A l l-n_{1}, n_{1}, l\right\rangle\left[n_{1}=n_{2} \wedge n_{1}=n_{3}\right]+\left\langle n_{1}, A l l-n_{2}, n_{2}, l\right\rangle\left[n_{1} \neq n_{2}\right]
\end{aligned}
$$

Also in this case, it holds $S C\left(t_{r_{1}}\right) \subseteq S M E\left(t_{r_{1}}\right)$, i.e., different instances of $t_{r_{1}}$ are potentially concurrent. As for $t_{r_{3}}$, there are no auto-conflicts.

We performed all the calculations with SNexpression and, on average, it took around a few dozen ms per test.

Detecting GTS rule instances which may run independently from each other would generally require more sophisticated calculations. Related concerns, however, are out of the scope of this paper.

\subsection{Checking Semiflows}

An opportunity offered by $\mathrm{SN}$ is the automated verification of structural invariants involving place marking ( $P$-invariants, or semi-flows) or transition sequences ( $T$-invariants). These invariants do not take account of inhibitor arcs and priorities. However, they allow you to formally and efficiently check interesting properties, orthogonal or complementary to structural relations. Focusing on $P$-invariants, we can distinguish between colored (symbolic) and numerical ones. The former give us both qualitative and quantitative information, hence are of particular interest.

The structural calculus implemented in SNExpression tool can be used to verify whether a $P$-indexed vector $\mathbf{i}$ of bag-expressions $\mathbf{i}[p]: \mathcal{D}(p) \rightarrow \operatorname{Bag}\left[\mathcal{D}_{\text {inv }}\right]$ is a coloured $P$-invariant. $\mathcal{D}_{i n v}$ is the invariant's color domain.

Vector $\mathbf{i}$ is a $P$-invariant (or semi-flow) if and only if, for each transition $t$, $\sum_{p \in P} \mathbf{i}[p] \circ(1 \cdot \mathrm{O}[p, t]-1 \cdot \mathrm{I}[p, t]) \equiv \epsilon^{6}$. The invariant expression we obtain from the SN initial marking is: $\sum_{p} \mathbf{i}[p]\left(\mathbf{m}_{0}[p]\right)$.

Consider the GTS composed of two simple rules shown in Figure 3 : one $\left(t_{r_{1}}\right)$ replaces everywhere edge label $l b_{1}$ with $l b_{2}$, the other $\left(t_{r_{2}}\right)$ deletes edge-loops whose label is neither $l b_{1}$ nor $l b_{2}$. In this fairly common case, the only non-null invariant entry refers to place Edge, which encodes the connected components

\footnotetext{
${ }^{6}$ negative terms in a linear combination impact on the final outcome
} 


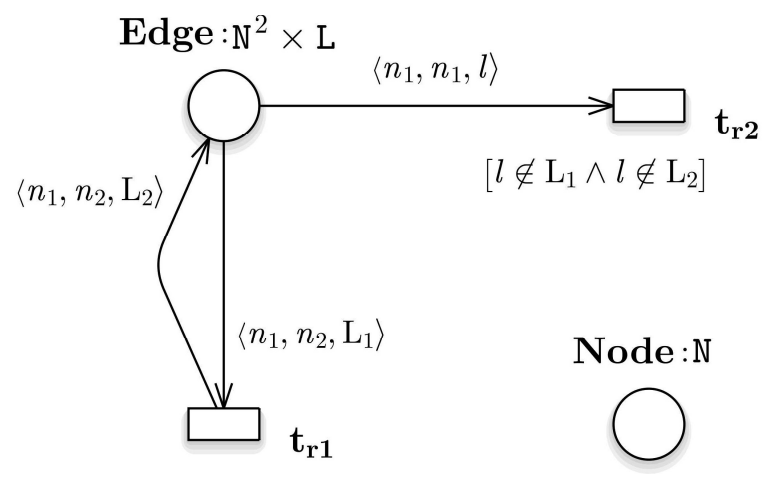

Figure 3: GTS composed of two rules

of a graph. Let $\mathrm{X}$ denote the neutral color, $x: \mathrm{X}, l: \mathrm{L}, n_{1}, n_{2}: \mathrm{N}$. Here are some possible invariants:

$$
\begin{array}{lr}
\text { pinv }_{1}:=\mathrm{N}^{2} \times \mathrm{L} \rightarrow \mathrm{X} & \langle x\rangle\left[l \in \mathrm{L}_{1} \vee l \in \mathrm{L}_{2}\right] \\
\text { pinv }_{2}:=\mathrm{N}^{2} \times \mathrm{L} \rightarrow \mathrm{N}^{2} & \left\langle n_{1}, n_{2}\right\rangle\left[n_{1} \neq n_{2}\right] \\
\text { pinv }_{3}:=\mathrm{N}^{2} \times \mathrm{L} \rightarrow \mathrm{N}^{2} \times \mathrm{L} & \left\langle n_{1}, n_{2}\right\rangle\left[l \in \mathrm{L}_{1} \vee l \in \mathrm{L}_{2}\right]
\end{array}
$$

The three expressions above satisfy the invariance property based on composition, therefore, are $P$-invariants. You can check it with SNExpression (which adopts a syntax very close to that used here) in just a few ms. Notice that these invariants depend on the size of subclasses $\mathrm{L}_{1}, \mathrm{~L}_{2}$ : the composition involving pinv $v_{1}$ and $t_{r_{1}}$, e.g., results in $\left|\mathrm{L}_{2}\right| \cdot\langle x\rangle-1 \cdot\left|\mathrm{L}_{1}\right| \cdot\langle x\rangle$, which is equal to $\epsilon$ if, as we are assuming, the two subclasses have the same size.

These invariants have a simple interpretation: pinv $v_{1}$ means that the total of edges with label $l b_{1}$ or $l b_{2}$ is preserved; pinv $v_{2}$ means that edges whose source differs from the target are preserved, without considering their labels; pinv $v_{3}$ is like pinv $v_{1}$ but more precise: it tells that edges with label $l b_{1}$ or $l b_{2}$, no matter which, are preserved.

\subsubsection{Numerical Semiflows}

Due to a property of SN arc functions, we may also check for numerical semiflows (or even minimal semiflow bases), typical of classical low-level PN. The size of a bag is the sum of multiplicities of the bag's elements.

Property 3 A SN arc-function $F: \mathcal{D}(t) \rightarrow \operatorname{Bag}[\mathcal{D}(p)]$ may be rewritten as $\sum_{i} \lambda_{i} \cdot T_{i}\left[g_{i}\right], \lambda_{i} \in \mathbb{N}$, where tuple guards are mutually exclusive and:

$$
\forall i \exists k \in \mathbb{N} \forall b \in \mathcal{D}(t): g_{i}(b)=\text { true } \Rightarrow\left|T_{i}(b)\right|=k
$$


In other words, any arc-function can be rewritten as a weighted sum of functiontuples that map to constant-size bags, but for colour instances making the tuple guards false. Consider $\left\langle\mathrm{C}_{i}-c\right\rangle$, which, depending on whether the colour bound to $c$ belongs to subclass $\mathrm{C}_{i}$ or not, has size $\left|\mathrm{C}_{i}\right|-1$ or $\left|\mathrm{C}_{i}\right|$ : we may rewrite $\left\langle\mathrm{C}_{i}-c\right\rangle \rightarrow\left\langle\mathrm{C}_{i}-c\right\rangle\left[c \in \mathrm{C}_{i}\right]+\left\langle\mathrm{C}_{i}\right\rangle\left[c \notin \mathrm{C}_{i}\right]$, according to Property 3 . This is the normal form used by SNexpression for bag-expressions.

As a consequence, by embedding function-tuple guards in transition guards (always possible), we can split any SN transition into an equivalent set of mutually-exclusive replica 7 whose arc-functions are "constant-size". In analogy with low-level PN, a $|P| \cdot|T|$ matrix $\mathbf{H}$ of $\mathbb{Z}$ values is thus defined, whose $[p, t]$ entry is $|\mathrm{O}[p, t](b)|-|\mathrm{I}[p, t](b)|$, for any $b \in \mathcal{D}(t)$. Any non-null $P$-vector $\mathbf{y}$ which is a positive integer solution of the matrix product $\mathbf{y} * \mathbf{H}=\mathbf{0}$ is a semiflow, expressing a conservative law for tokens flowing through the places corresponding to semiflows's non-zero entries, which abstracts from tokens' color. We say a place $p$ is covered by a semiflow is the semiflow's $p$-entry is non-null. We say an SN is covered if every place is. One such an SN is color-safe.

As for the GTS example of this section, place Edge is not covered by numerical semi-flows. We shall exploit (numerical) semiflows in the next section.

\section{SN Composite Graph Transformation Rules}

In this section, we define a more structured type of GTR able to emulate complex graph transformations (some of which) peculiar to algebraic, rule-based models. We use SN subnets with a well-defined layout.

Until now, we have considered context-dependent graph transformations. Some typical operations on graphs, however, apply in undetermined contexts: an example treated here is node deletion with simultaneous removal of incident edges, natively supported by the single-pushout [29] approach, but not by the DPO. Other examples, considered later, are node merge/split.

It is not possible to represent these operations on graphs through a single SN transition, due to the low data abstraction provided by SN arc-function syntax. Using the All function is not the solution, since it yields all the elements of a given color class.

The workaround consists of representing a GTR through an SN subnet, called composite rule, composed of one observable transition, which operates context-dependent changes and triggers a finite sequence of immediate transition instances in charge of performing changes in unspecified contexts.

Composite rules meet a quite simple structural pattern making it possible their efficient validation. Some subnet places have a neutral domain, their incident weight-one arcs do not carry any annotation, for simplicity (it should be $\langle A l l\rangle)$. We denote with ${ }^{\bullet} t, t^{\bullet}$, the set of places linked to a transition $t$ through non-null input, output arc-functions, respectively.

\footnotetext{
${ }^{7}$ that is, $t$ may be equivalently "split" into a set $E Q=\left\{t_{i}\right\}$, such that $\mathcal{D}(t)=\mathcal{D}\left(t_{i}\right)$, $\forall t_{i} \in E Q$, and $\mathbf{m}[t, b\rangle \mathbf{m}^{\prime} \Leftrightarrow \exists t_{i} \in E Q: \mathbf{m}\left[t_{i}, b\right\rangle \mathbf{m}^{\prime}, \forall \mathbf{m}, b \in \mathcal{D}(t)$
} 
Definition 4 (SN Composite GTR) A composite rule is a SN including, among the others, places Edge, Node (whose color domains have been previously defined), neutral places Start, Trigger, one observable transition $t_{r}$, and a set Imm of immediate transitions including end, such that:

- Start $\in$ end $\boldsymbol{\bullet}^{\bullet} \bullet_{r} ;$ Trigger $\in t_{r}{ }^{\bullet}$

- $\forall t \in \operatorname{Imm}:$ Trigger $\epsilon^{\bullet} t \quad \forall t \in \operatorname{Imm}-\{e n d\}:$ Trigger $\in t^{\bullet}$

- $\forall t \in \operatorname{Imm}-\{e n d\}: t S M E$ end

- the SN is covered by (numerical) semiflows

- every SN transition satisfies Lemma 1

Places Start, Trigger are covered by a semi-flow, by construction. The coverage requirement for the other places is to ensure color-safeness. The mutual exclusion between end and any other immediate transition guarantees that the rule's behaviour is well-defined. Whenever end fires, it disables all the other immediate transitions, bringing back the $\mathrm{SN}$ to its initial state.

As an example, Figure 4 shows an SN composite rule deleting a graph node without successors but itself, together with all incident edges. Transition $t_{r}$ checks for the application condition by matching a node $v_{i}$, then transition delInEdge removes all edges with target $v_{i}$, finally end deletes $v_{i}$ by respecting the dangling-edge condition. The only extra place NoSucc, initially marked with any class $\mathrm{N}$ colour, is clearly covered by a semiflow. The mutual exclusion between end and delInEdge is ensured by the arc-function $\mathrm{H}$ [Edge, end].

We need to slightly change the notion of GTS to include composite rules.

Definition 5 (Generalized SN Graph Transformation System) Let $G_{0}$ be a graph, $\mathcal{R}_{e}$ be a set of (SN) Graph Transformation Rules (Definition 2) and $\mathcal{R}_{c}$ a set of disjoint SN implementing composite rules (Definition 4). The associated GTS is the SN model obtained by sharing places Node and Edge among $\mathcal{R}_{e}$ and $\mathcal{R}_{c}$, with $\mathbf{m}_{0}$ [Node], $\mathbf{m}_{0}$ [Edge] encoding $G_{0}$, and $\left\{\operatorname{Start}_{i}\right\}$ places of $\mathcal{R}_{c}$ initially holding one neutral token.

The SN Reachability Graph (precisely, its projection on places Node and Edge) defines the GTS state-transition system.

\section{A Comparison with the DPO Approach}

In the second part of the paper, we compare the SN-based graph transformation approach with a classical, rule-based one. We consider basic graph rewriting, in particular, the algebraic approach based on double-pushout (DPO), one of the most well-known (Turing-complete) approaches. We do not use the original, categorical pushout concepts, but we follow a somewhat operational 


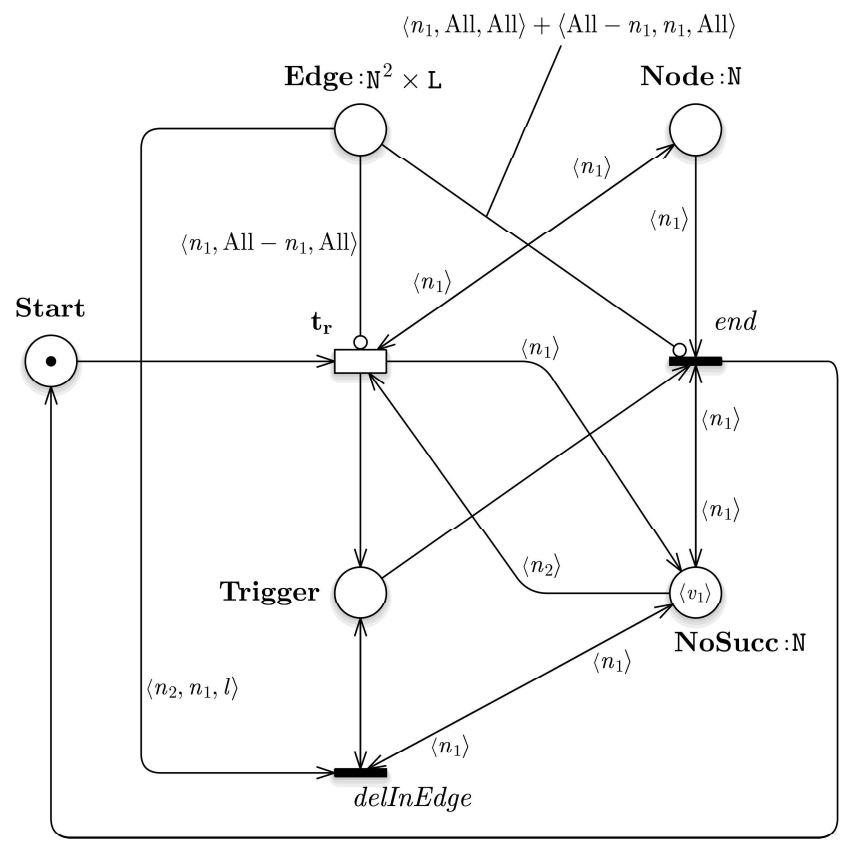

Figure 4: Composite rule: removal of a node without proper successors

line. We use well-known definitions which solely refer to the concepts of graph (total) morphism and gluing, formally presented in the background.

The aim is twofold: to provide the SN-based graph transformation approach with a theoretically sound semantic characterization, and to promote interoperability among different modelling formalisms and tools.

We follow a constructive approach: given a DPO rule, we show how to formally derive (step-by-step) the color annotations of the corresponding SN GTR, first considering "injective" DPO rules, then non-injective ones.

After recalling the base concepts of DPO rules (Section 7.1), we formalize the translation of injective rules in the (widespread) case of an injective match of rules (Section 7.2). Then we set a precise relationship between rule matches and SN transition instances, also considering non-injective matches (Section 7.3). Finally, we deal with non-injective DPO rules (Section 7.4) and shortly discuss on the reverse direction $\mathrm{SN} \rightarrow \mathrm{DPO}$ (Section 7.5).

\subsection{Basic Definitions}

DPO Graph Transformation Rule. A DPO rule $r$ is composed of three graphs, $L, I, R$ (left-hand side, interface, and right-hand side, respectively) and two morphisms: $L \stackrel{\phi_{L}}{\longleftarrow} I \stackrel{\phi_{R}}{\longrightarrow} R$.

The elements of graph $L$ not belonging to the image of $\phi_{L}$ are said obsolete, whereas the elements of $R$ not in the image of $\phi_{R}$ are said fresh. If both 
morphisms are injective we say that the rule is injective.

The application of an injective rule has an intuitive explanation: once a match of the rule's left-hand side is found in a host graph $G$, the rule applies by removing obsolete nodes/edges, adding fresh elements, and preserving $I$, which plays the role of a solid attachment point.

However, if $\phi_{L}$ or $\phi_{R}$ is not injective, the previous interpretation has to be corrected because of some merge/split effect (depending on whether $\phi_{R}$ or $\phi_{L}$ is non-injective, respectively). The general semantics of graph transformation makes use of the graph gluing concept.

Graph Transformation. Given $r=\left(L \stackrel{\phi_{L}}{\longleftarrow} I \stackrel{\phi_{R}}{\longrightarrow} R\right)$, a graph $G$ is transformed by $r$ into a graph $H$ (written $G \stackrel{r}{\Rightarrow} H$ ) if there is a graph $C$ (context) and a morphism $\nu: I \rightarrow C$, such that $G \cong L+_{\phi_{L}, \nu} C$ and $H \cong R+_{\phi_{R}, \nu} C$.

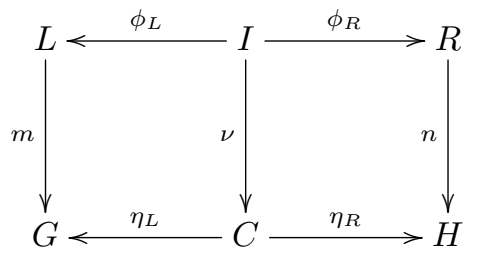

We illustrate this on the commutative diagram above, where the morphisms $m$ and $n$ are called match and co-match, respectively. In other words, we search for an unknown graph $C$ (called context) such that the host graph $G$ is the gluing of $L$ and $C$ over $I$. If such a context does exist, we rewrite $G$ to the graph obtained by gluing $C$ and $R$ over $I$.

Many papers make strong assumptions on rules. Most of them deal with injective rules and extend this assumption to rule match. Almost all assume that the morphism $\phi_{L}$ is injective. We shall first consider the base case of an injective rule/match then we shall treat non-injective $m$ and $\phi_{R}$.

Differently from term rewriting, the existence of a match of the left-hand side of a rule in a host graph doesn't ensure that the rule can apply. Two more conditions guarantee it, the dangling-edge condition (if we remove a node, then we contextually remove all incident edges) and the identification condition (a match may only identify elements which are preserved). We call the sum of these two conditions gluing condition.

Property 4 Let $r=\left(L \stackrel{\phi_{L}}{\longleftarrow} I \stackrel{\phi_{R}}{\longrightarrow} R\right)$ be a rule and $m: L \rightarrow G$ be a graph morphism, such that $m_{E}$ is injective. Then a context $C$ and a morphism $\nu$ : $I \rightarrow C$ such that $G \cong L+_{\phi_{L}, \nu} C$ do exist if and only if

- $\forall v \in N_{L}$ such that $m(v)$ is incident to $e \in E_{G}-m_{E}\left(E_{L}\right): v \in \phi_{L}\left(N_{I}\right)$

- $\forall v_{1}, v_{2} \in N_{L}, v_{1} \neq v_{2}: m\left(v_{1}\right)=m\left(v_{2}\right) \Rightarrow v_{1}, v_{2} \in \phi_{L}\left(N_{I}\right)$

Informally, the dangling condition (the 1st one) says that every node of $L$ whose image is incident to an edge of $G$ which is not in the image of $m$ is not obsolete. 
If $\phi_{L}$ is injective and $m$ satisfies the gluing condition, the context $C$ and the morphism $\nu$ are unique. Rule $r$ thus applies deterministically (up to graph isomorphism) and we use the notation $G \stackrel{r, m}{\longrightarrow} H$.

Figure 5 gives an example of injective DPO rule and corresponding graph transformation. We shall refer to it to illustrate some basic concepts/notations.

By convention, we describe a morphism $f: G_{1} \rightarrow G_{2}$ by associating identifiers 1 through $\left|N_{G_{1}}\right|$ with nodes of the source graph (we may thus speak of $i^{t h}$ node), and pair-wise disjoint, non-empty sets of values in the same range to nodes belonging to the image of $G_{1}$. In the case of injective morphism, all these sets are singletons.

\subsection{Mapping Injective DPO Rules to SN Graph Transformation Rules.}

Let $t_{r}$ denote the transition corresponding to $r=L \stackrel{\phi_{L}}{\longleftarrow} I \stackrel{\phi_{R}}{\longrightarrow} R$. The following steps formalize the functions on the arcs connecting $t_{r}$ to places Node, Edge and the guard of $t_{r}$. In the sequel, $\bigwedge_{\emptyset}(\ldots) \equiv$ true, whereas $\sum_{\emptyset}(\ldots) \equiv \epsilon$.

\section{Procedure 1 (SN translation of an injective DPO rule - injective match)}

1. The set $\operatorname{Var}\left(t_{r}\right)=\left\{n_{i}, i: 1 \ldots \mathrm{k}\right\}, k \in \mathbb{N}^{+}$, of type- $\mathrm{N}$ variables (projections) used in arc functions is partitioned into:

$$
\operatorname{Var}\left(t_{r}\right)=\operatorname{Var}_{I} \cup \operatorname{Var}_{\text {obs }} \cup \operatorname{Var}_{\text {fresh }}
$$

$\operatorname{Var}_{I}=\left\{n_{i}, i: 1 \ldots\left|N_{I}\right|\right\}$ corresponds to the set of interface's nodes, whereas $V a r_{o b s}$ and $V a r_{\text {fresh }}$ are isomorphic to $N_{L}-\phi_{L}\left(N_{I}\right)$ and $N_{R}-\phi_{R}\left(N_{I}\right)$, respectively (the obsolete and fresh nodes). By convention, if $n_{i} \in V a r_{o b s}$ and $n_{j} \in \operatorname{Var}_{\text {fresh }}$, then $i<j$. Let var $: N_{I} \cup N_{L} \cup N_{r} \rightarrow \operatorname{Var}\left(t_{r}\right)$ map each graph node to the corresponding variable: $\operatorname{var}\left(N_{I}\right)=\operatorname{var}\left(\phi_{L}\left(N_{I}\right)\right)=\operatorname{var}\left(\phi_{R}\left(N_{I}\right)\right)$.

2. The arc functions involving place Node are

$$
\begin{aligned}
\mathrm{I}\left[\text { Node }, t_{r}\right] & =\sum_{n_{i} \in \operatorname{Var}_{I} \cup V a r_{\text {obs }}}\left\langle n_{i}\right\rangle \quad \mathrm{O}\left[\text { Node }, t_{r}\right]=\sum_{n_{i} \in \operatorname{Var}_{I} \cup V a r_{\text {fresh }}}\left\langle n_{i}\right\rangle \\
\mathrm{H}\left[\text { Node }, t_{r}\right] & =\sum_{n_{i} \in \operatorname{Var}_{\text {fresh }}}\left\langle n_{i}\right\rangle
\end{aligned}
$$

3. Having mapped graph nodes to transition's variables, each $e \in E_{I} \cup E_{L} \cup E_{R}$, with $l(e)=l b_{k}$, is consequently identified by a tuple $\left\langle n_{i}, n_{j}, \mathrm{~L}_{k}\right\rangle$, where $n_{i}=$ $\operatorname{var}(s(e)), n_{j}=\operatorname{var}(t(e))$. Since we are considering multi-graphs, we can represent the sets $E_{I}, E_{L}, E_{R}$ as (symbolic) bag: $\AA^{8} \operatorname{bag}_{E_{I}} \in B a g\left[\operatorname{Var}_{I}^{2} \times \mathrm{L}\right]$, $\operatorname{bag}_{E_{L}} \in \operatorname{Bag}\left[\left(\operatorname{Var}_{I} \cup \operatorname{Var}_{\text {obs }}\right)^{2} \times \mathrm{L}\right], \operatorname{bag}_{E_{R}} \in \operatorname{Bag}\left[\left(\operatorname{Var}_{I} \cup \operatorname{Var}_{\text {fresh }}\right)^{2} \times \mathrm{L}\right]$. Since $\phi_{R}$ and $\phi_{L}$ are injective morphisms, $\operatorname{bag}_{E_{I}} \leq \operatorname{bag}_{E_{L}} \wedge \operatorname{bag}_{E_{I}} \leq \operatorname{bag}_{E_{R}}$.

\footnotetext{
${ }^{8}$ They can be seen both as formal bags of functions and bag-functions
} 
4. The arc functions involving place Edge are:

$$
\begin{array}{ll}
\mathrm{I}\left[\text { Edge }, t_{r}\right]=b a g_{E_{L}} & \mathrm{O}\left[\text { Edge, } t_{r}\right]=b a g_{E_{R}} \\
\mathrm{H}\left[\text { Edge, } t_{r}\right]=H_{1} \quad(\text { see Lemma 1, C 3. }) &
\end{array}
$$

5. The guard $g\left(t_{r}\right)$ is a conjunctive form which contains the following predicates:

$$
\begin{aligned}
& \forall n_{i}, n_{j} \in \operatorname{Var}_{\text {fresh }}, i \neq j: n_{i} \neq n_{j} \\
& \forall n_{i} \in \operatorname{Var}_{\text {obs }} \forall n_{j} \in \operatorname{Var}_{\text {obs }} \cup \operatorname{Var}_{I}, i \neq j: n_{i} \neq n_{j}
\end{aligned}
$$

We have mechanically obtained the SN in Figure 6 (a) from the DPO rule in Figure 5, by applying Procedure 1. In this case, we have: $\operatorname{Var}_{I}=\left\{n_{1}, n_{2}\right\}$, $V a r_{o b s}=\emptyset, V_{\text {fresh }}=\left\{n_{3}\right\}$.

Property 5 A transition $t_{r}$ defined according to Procedure 1 is a SN Graph Transformation Rule (Definition 2).

Proof. The 1st condition of Lemma 1 holds since $\left(\sum_{n_{i} \in \operatorname{Var}_{\text {fresh }}}\left\langle n_{i}\right\rangle\right)\left[g\left(t_{r}\right)\right] \leq$ $\langle A l l\rangle$ and $\mathrm{W}^{+}\left[\right.$Node, $\left.t_{r}\right]=\left(\sum_{n_{i} \in V_{\text {ar }} \text { fresh }}\left\langle n_{i}\right\rangle\right)\left[g\left(t_{r}\right)\right]-\ldots$ As for the 2nd condition, it is sufficient to observe that $\left\langle n_{1}+n_{2}\right\rangle \circ \mathrm{O}\left[\right.$ Edge, $\left.t_{r}\right] \subseteq \operatorname{Var}_{I} \cup \operatorname{Var}_{\text {fresh }}$ $=\overline{\mathrm{O}\left[\text { Node }, t_{r}\right]}$. The $3 \mathrm{rd}$ and last condition is enforced by step 4 .

Enabled instances of transition $t_{r}$ meet the property below 9

Property 6 Let $t_{r}$ be the SN transition obtained by translating an injective DPO rule $r$ according to Procedure 1 and $\mathbf{m}$ a graph-encoding.

If $\left(t_{r}, b\right)$ is enabled in $\mathbf{m}$ then $\forall n_{i}, n_{j} \in \operatorname{Var}\left(t_{r}\right), i \neq j: n_{i}(b) \neq n_{j}(b)$.

Proof. Since $\mathbf{m}$ [Node] is type-set, due to I[Node, $\left.t_{r}\right], \mathrm{H}\left[\right.$ Node, $\left.t_{r}\right]$ (Step 3), enabled instances of $t_{r}$ verify, other than $g\left(t_{r}\right)$ (Step 5), these implicit predicates:

$$
\begin{aligned}
& \forall n_{i}, n_{j} \in \operatorname{Var}_{I}, i \neq j: n_{i}(b) \neq n_{j}(b) \\
& \forall n_{i} \in \operatorname{Var}_{\text {fresh }} \forall n_{j} \in \operatorname{Var}_{I} \cup \operatorname{Var}_{\text {obs }}: n_{i}(b) \neq n_{j}(b) .
\end{aligned}
$$

Sometimes, we may rewrite the color annotations of the SN transition resulting from Procedure 1 in a somewhat simpler form.

Hereinafter, we shall use two extra symbols: $\operatorname{Var}_{I}^{*} \subseteq \operatorname{Var}_{I}$ includes all and only $\operatorname{Var}_{I}$ variables occurring on I Edge, $\left.t_{r}\right]$ whereas $\operatorname{Var}_{I}^{-}=\operatorname{Var}_{I}-\operatorname{Var}_{I}^{*}$.

\footnotetext{
${ }^{9}$ Given a transition instance $b$, we may equivalently write $b:\left(n_{i}=v_{i}, \ldots\right)$, or $n_{i}(b)=v_{i}$, depending on whether we see $n_{i}$ as a variable or a projection
} 
Property 7 Let $t_{r}$ be the SN translation of an injective rule $r=L \stackrel{\phi_{L}}{\longleftarrow} I \stackrel{\phi_{R}}{\longrightarrow} R$, according to Procedure 1. If we erase $\operatorname{Var}_{I}^{*}$ from I[Node, $\left.t_{r}\right]$, O[Node, $\left.t_{r}\right]$, and introduce in $g\left(t_{r}\right)$ the predicates $n_{i} \neq n_{j}, \forall n_{i} \in \operatorname{Var}_{I}^{*}, n_{j} \in \operatorname{Var}_{I}, i \neq j$, we get an equivalent GTR.

Proof. Let $t_{r}^{\prime}$ be obtained by rewriting $t_{r}$, as indicated. By construction, $t_{r}^{\prime}$ has the same color domain as $t_{r}$, and $\mathrm{W}^{+}\left[p, t_{r}\right]=\mathrm{W}^{+}\left[p, t_{r}^{\prime}\right]$, i.e., any two instances $\left(t_{r}, b\right)$ and $\left(t_{r}^{\prime}, b\right)$, when firing, have the same effect. If $\left(t_{r}, b\right)$ is enabled, then also $\left(t_{r}^{\prime}, b\right)$ is, based on the aforementioned implicit predicates (Property 6). On the other side, if $\left(t_{r}^{\prime}, b\right)$ is enabled in $\mathbf{m}$ (therefore, I[Edge, $\left.t_{r}^{\prime}\right](b) \leq \mathbf{m}[$ Edge $]$ ), we know that $F(b)=\sum_{n_{i} \in \operatorname{Var}_{I}^{*}} n_{i}(b) \leq\left\langle n_{1}+n_{2}\right\rangle \circ \mathbf{m}[$ Edge $] \leq \mathbf{m}$ [Node] (Definition 1. graph-encoding), with $F(b)$ being type-set due to the guard. Given that $\mathrm{I}\left[\right.$ Node, $\left.t_{r}\right](b)$ is equal to the disjoint sum I[Node, $\left.t_{r}^{\prime}\right](b)+F(b)$ (both terms are less than or equal to $\mathbf{m}[$ Node] $),\left(t_{r}, b\right)$ is enabled in $\mathbf{m}$.

Figure 6 (b) shows the equivalent (simpler) translation of DPO rule in Figure 5. according to Property 7. In this case, $\operatorname{Var}_{I}^{*}=\left\{n_{1}, n_{2}\right\}$.

An example of mechanical translation of an injective DPO rule involving node removal, according to Procedure 1, is shown in Figures 78. In that case, we have: $\operatorname{Var}_{I}=\operatorname{Var}_{I}^{*}=\{n 1\}, \operatorname{Var}_{\text {obs }}=n_{2}, \operatorname{Var}_{\text {fresh }}=\emptyset$. You can see that the inhibitor arc function ensuring the dangling-edge condition takes account of the (only) edge incident to the removed node, which is contextually withdrawn by the rule. This corresponds to the weight-two term in the arc-function expression.

We may carry out a comparison between an injective $r: L \stackrel{\phi_{L}}{\longleftarrow} I \stackrel{\phi_{R}}{\longrightarrow} R$ and the corresponding SN transition $t_{r}$ through the diagram below, which obscures, however, the role played by inhibitor arcs and transition guard:

$$
\left(\begin{array}{c}
\mathrm{I}\left[\text { Node }, t_{r}\right] \\
\operatorname{bag}_{E_{L}}
\end{array}\right) \geq\left(\begin{array}{c}
\mathrm{I}\left[\text { Node }, t_{r}\right] \cap \mathrm{O}\left[\text { Node }, t_{r}\right] \\
\operatorname{bag}_{E_{I}}
\end{array}\right) \leq\left(\begin{array}{c}
\mathrm{O}\left[\text { Node }, t_{r}\right] \\
\operatorname{bag}_{E_{R}}
\end{array}\right)
$$

\subsection{Rule Match and Graph Transformation}

In order to define the semantics of a transition $t_{r}$ representing a DPO rule $r$, we need to formalize a graph morphism $m: L \rightarrow G$ (hereinafter, simply match) in terms of $t_{r}$. We first consider injective matches, to which we have so far implicitly referred. Then, we treat the more general, even if less frequent, case.

We set an intuitive relationship between $m: L \rightarrow G$ and an equivalence class of instances of $t_{r}$, holding independently on whether $m$ is injective or not. Let $b_{1}, b_{2} \in \mathcal{D}\left(t_{r}\right)=\mathrm{N}^{\left|\operatorname{Var}_{I}\right|+\left|\operatorname{Var}_{o b s}\right|+\left|\operatorname{Var}_{f r e s h}\right|}$ : we write $b_{1} \equiv b_{2}$ if and only if $\forall n_{i} \in \operatorname{Var}_{I} \cup V a r_{o b s}: n_{i}\left(b_{1}\right)=n_{i}\left(b_{2}\right)$.

In a graph encoding context, equivalent instances behave in the same way. 


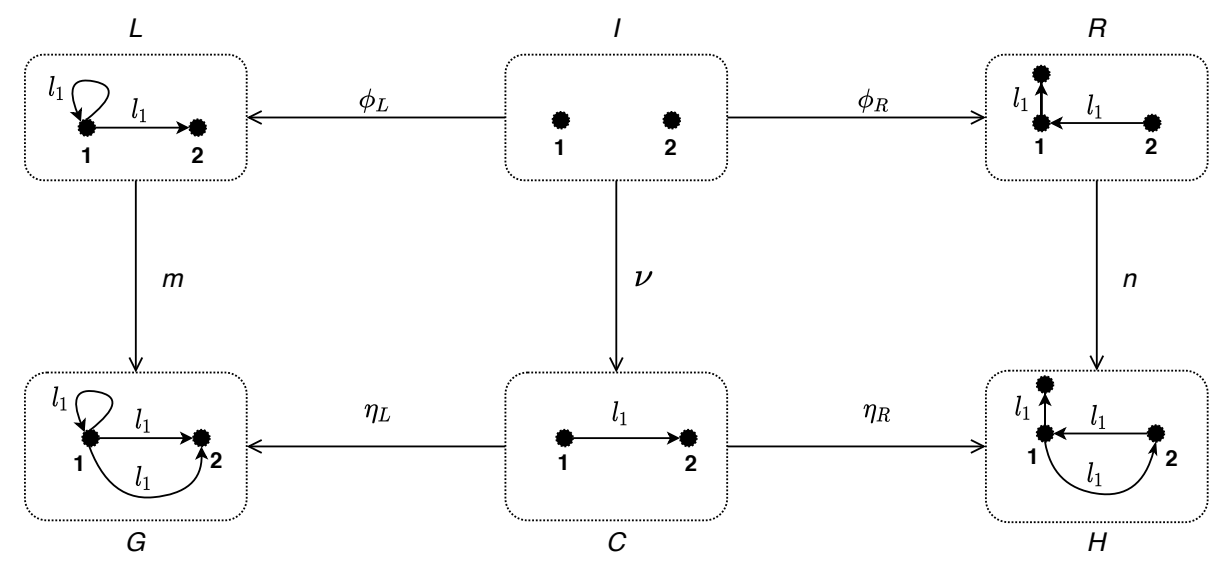

Figure 5: Injective DPO rule and graph transformation

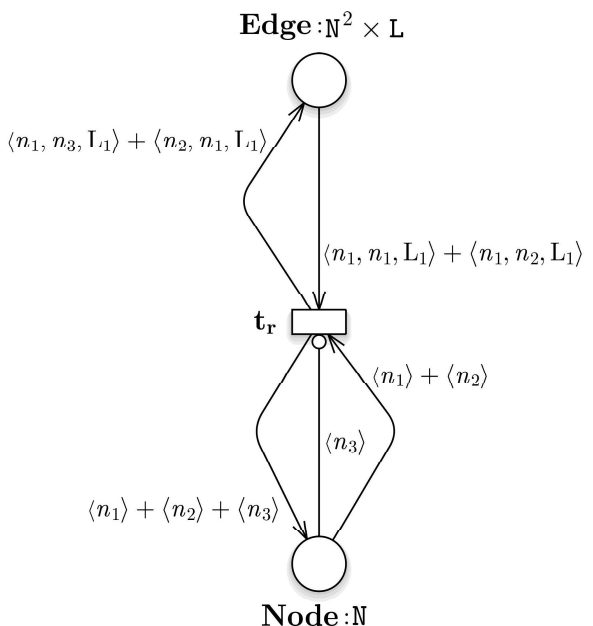

(a) Original

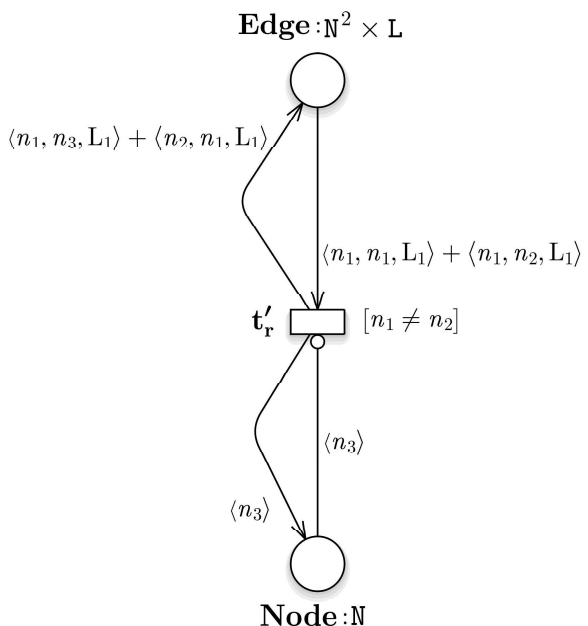

(b) Equivalent

Figure 6: SN Translation(s) of DPO rule in Figure 5 
Property 8 Let $t_{r}$ be the SN transition translating an injective DPO rule $r$, $b_{1}, b_{2} \in \mathcal{D}\left(t_{r}\right)$, with $b_{1} \equiv b_{2}$, and $\mathbf{m}_{G}$ a graph-encoding.

If $\mathbf{m}_{G}\left[t_{r}, b_{1}\right\rangle \mathbf{m}_{H}$ then $\mathbf{m}_{G}\left[t_{r}, b_{2}\right\rangle \mathbf{m}_{H}^{\prime}$, with $H \cong H^{\prime}$.

Proof. It stems from the fact that $b_{1}, b_{2}$ (by definition of $\equiv$ ) are one the colorpermutation of the other.

As explained (Procedure 1), the left-hand side $L$ of an injective rule $r$ is isomorphic to a graph whose nodes are $N_{L}=\operatorname{Var}_{I} \cup \operatorname{Var}_{\text {obs }}$ (the $i^{t h}$ node of $L$ is identified by $n_{i}$ ), and whose edges are represented by the symbolic bag $\operatorname{bag}_{E_{L}}$. Precisely, we define the edge set $E_{L}$ as: $\forall T=\left\langle n_{i}, n_{j}, \mathrm{~L}_{k}\right\rangle \in \operatorname{bag}_{E_{L}}$, there are $b_{a g} g_{E_{L}}(T)$ edges $e \in E_{L}$, such that $s(e)=n_{i}, t(e)=n_{j}, l(e)=\mathrm{L}_{k}$. The definition below meets this convention.

Definition 6 (match and corresponding instance) Let $r=L \stackrel{\phi_{L}}{\longleftarrow} I \stackrel{\phi_{R}}{\longrightarrow} R$ be an injective DPO rule, $t_{r}$ its SN translation, $G$ a graph, and $\mathbf{m}_{G}$ its encoding.

Given $m: L \rightarrow G$, we say that $b_{m} \in \mathcal{D}\left(t_{r}\right)$ is a corresponding instance if and only if $\forall n_{i} \in \operatorname{Var}_{I} \cup \operatorname{Var} r_{o b s}: b_{m}\left(n_{i}\right)=\operatorname{col}_{G}\left(m_{N}\left(n_{i}\right)\right)$, where $\operatorname{col}_{G}$ is the color-map used in $\mathbf{m}_{G}$. The class of corresponding instances is $\left[b_{m}\right]_{\equiv}$.

The other way round, let $b \in \mathcal{D}\left(t_{r}\right)$, such that $\mathrm{I}\left[\right.$ Node, $\left.t_{r}\right](b) \leq \mathbf{m}_{G}[$ Node $] \wedge$ $\mathrm{I}\left[\right.$ Edge, $\left.t_{r}\right](b) \leq \mathbf{m}_{G}[$ Edge $]$. The corresponding morphism $m_{b}: L \rightarrow G$ is:

- $\forall n_{i} \in \operatorname{Var}_{I} \cup \operatorname{Var}_{o b s}: m_{b_{N}}\left(n_{i}\right)=\operatorname{col}_{G}^{-1}\left(n_{i}(b)\right)$

- $\forall e \in E_{L}: m_{b_{E}}(e)=e^{\prime} \in E_{G}$, with $s\left(e^{\prime}\right)=m_{b_{N}}(s(e)), t\left(e^{\prime}\right)=m_{b_{N}}(t(e))$, $l\left(e^{\prime}\right)=l(e)$

In the 2nd part of Definition 6, the assumption on the input functions ensures that the colors bound to any $n_{i} \in \operatorname{Var}_{I} \cup V a r_{\text {obs }}$ correspond to (describe) nodes of $G$ (analogously for color-tuples describing edges). That is, the transition instance "matches" a graph morphism.

Definition 6 thus sets a bijection between morphisms matching an injective DPO rule $r$ to a graph $G$ and (equivalence classes of) instances of the transition $t_{r}$ (the SN translation of $r$ ) verifying the input enabling condition in $\mathbf{m}_{G}$. For simplicity, symbol $b_{m}$ will denote a representative of the class of instances corresponding to a given match $m$.

The following lemma precisely states the semantics of the SN translation of an injective DPO rule.

Lemma 2 Let $r=L \stackrel{\phi_{L}}{\longleftarrow} I \stackrel{\phi_{R}}{\longrightarrow} R$ be an injective rule, $t_{r}$ its $S N$ translation, and $G$ a graph.

$m: L \rightarrow G$ satisfies the gluing condition (Property 4) if and only if $\left(t_{r}, b_{m}\right.$ ) is enabled in $\mathbf{m}_{G}$. 


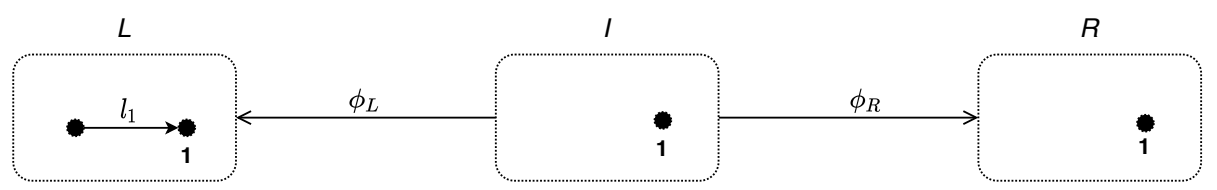

Figure 7: DPO rule removing a (source) node from a graph

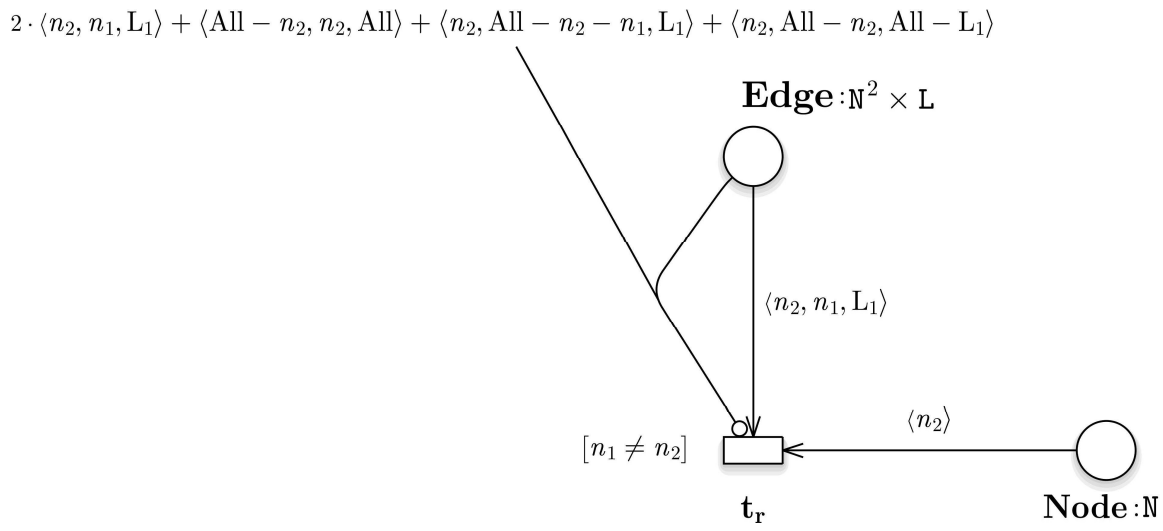

Figure 8: SN translation of rule in Figure 7

Proof. Consider $\Rightarrow$. We assume that $m$ is injective. Since $n_{i}\left(b_{m}\right) \neq n_{j}\left(b_{m}\right)$, $\forall n_{i}, n_{j} \in \operatorname{Var}_{I} \cup V a r_{o b s}, i \neq j$ (i.e., $n_{i}, n_{j}$ are bound to different colors), it holds $\mathrm{I}\left[\right.$ Node, $\left.t_{r}\right]\left(b_{m}\right) \leq \mathbf{m}_{G}\left[\right.$ Node]. By the way, $\mathrm{I}\left[\right.$ Edge, $\left.t_{r}\right]\left(b_{m}\right) \leq \mathbf{m}_{G}[$ Edge $]$ because $m$ is a morphism. We have just to consider inhibitor functions. Assume, by contradiction, that $\exists e: \mathrm{H}\left[\mathrm{Edge}, t_{r}\right]\left(b_{m}\right)(e) \leq \mathbf{m}_{G}[\mathrm{Edge}](e)$; due to the definition of $\mathrm{H}\left[\right.$ Edge, $\left.t_{r}\right]$, this is only possible if there is $n_{i} \in V a r_{o b s}$ such that, letting $v_{j}=n_{i}\left(b_{m}\right)$ be the associated colour (obsolete node), edge $e$ is incident to $v_{j}$ and there are more instances of $e$ than those which are withdrawn (better, required by the input arc function, whose image corresponds to the image of $m$ ). This would imply that there is an arc (an occurrence of $e$ ) incident to an obsolete node, which is not in the image of $m_{E}$, in contrast with the Hp (Property 4. dangling condition). As for $\mathrm{H}\left[\right.$ Node, $\left.t_{r}\right]$, we only have to chose colors for fresh variables such that $\forall n_{i}, n_{j} \in \operatorname{Var}_{\text {fresh }}, i \neq j, \forall n_{h} \in \operatorname{Var}_{I} \cup \operatorname{Var}_{\text {obs }}: n_{i}\left(b_{m}\right) \neq$ $n_{j}\left(b_{m}\right) \wedge n_{i}\left(b_{m}\right) \neq n_{h}\left(b_{m}\right) \wedge n_{j}\left(b_{m}\right) \neq n_{h}\left(b_{m}\right)$ : this is surely possible, because we assume color class $\mathrm{N}$ large enough (all these instances are isomorphic).

Consider $\Leftarrow$. We have to show that, if $\left(t_{r}, b\right)$ is enabled in $\mathbf{m}_{G}$, then $m_{b}$ verifies Property 4. The identification condition is trivially met: since I[Node, $\left.t_{r}\right](b) \leq$ $\mathbf{m}_{G}$ [Node], it holds: $n_{i}(b) \neq n_{j}(b), \forall n_{i}, n_{j} \in \operatorname{Var}_{I} \cup V a r_{o b s}, i \neq j$. As for the dangling condition, we can use pretty the same reasoning as for the 1st assertion. By definition of $\mathrm{H}\left[\right.$ Edge, $\left.t_{r}\right]$, we know that, if $n_{i} \in V a r_{o b s}, n_{i}(b)=v_{j}$, and a type-edge color tuple $e \in \mathbf{m}_{G}[\mathrm{Edge}]$ is "incident" to (contains) $v_{j}$, then there 
are just as many occurrences of $e$ in $\mathbf{m}_{G}$ [Edge] as those withdrawn by the firing of $b\left(\mathrm{I}\left[\right.\right.$ Edge,$\left.t_{r}\right](b)(e)=\mathbf{m}_{G}[$ Edge $\left.](e)\right)$ : that is, each edge of $G$ incident to the image $m_{b}\left(v_{j}\right)$ of an obsolete node $v_{j}$ of $L$ is in turn in the image of $m_{b}$.

The parallelism between DPO rules and their SN translation is complete observing that, given a rule $r=L \stackrel{\phi_{L}}{\longleftarrow} I \stackrel{\phi_{R}}{\longrightarrow} R$ and a match $m: L \rightarrow G$ satisfying the gluing condition, the application of $r$ and the firing of any corresponding instance of rule's translation $t_{r}$ have the same effect. This directly follows from the next explanation of a DPO rule's application, which is valid independently on whether $m$ and $\phi_{R}$ are injective or not:

1. we remove the image of obsolete elements, $m\left(L-\phi_{L}(I)\right)$, from $G$, to get the context graph $Q^{10}$, we directly derive morphism $\nu$ from $m$

2. we derive the target graph $H$ by gluing $C$ and $R$ over $I\left(H \cong R+{ }_{\phi_{r}, \nu} C\right)$ : we merge elements of $R, C$ with a common pre-image in $I$, whereas we include in $H$ all the other elements of $R$ and $C$, individually taken.

If $\phi_{R}$ is injective we do not perform any merge. In such a case, if $m$ is an injective match (we shall relax this condition) satisfying the gluing condition, and $b_{m}$ is a representative of corresponding instances of $t_{r}$, then we can reproduce the two steps above in terms of $\mathrm{SN}$ firing rule (functions $\mathrm{W}^{-}[p, t], \mathrm{W}^{+}[p, t]$ are defined in Table 11. Let $p \in\{$ Node, Edge $\}$ :

1. $\mathbf{m}_{G}[p]-\mathrm{W}^{-}\left[p, t_{r}\right]\left(b_{m}\right)=\mathbf{m}_{C}[p]$

2. $\mathbf{m}_{C}[p]+\mathrm{W}^{+}\left[p, t_{r}\right]\left(b_{m}\right)=\mathbf{m}_{H}[p]$

Corollary 1 (of Lemma 2) Let $r=L \stackrel{\phi_{L}}{\longleftarrow} \stackrel{\phi_{R}}{\longrightarrow} R$ be an injective DPO rule, $t_{r}$ its $S N$ translation, and $G$ a graph.

$m: L \rightarrow G$ is a match satisfying the gluing condition such that $G \stackrel{r, m}{\Longrightarrow} H$ if and only if $\mathbf{m}_{G}\left[t_{r}, b_{m}\right\rangle \mathbf{m}_{G^{\prime}}$, with $H \cong G^{\prime}$.

\subsubsection{Non-Injective Match}

The translation of an injective DPO rule $r$ into a single SN transition $t_{r}$, formalized by Procedure 1, only deals with injective matches of rule's left-hand side $L$ to a host graph $G$. If we want to include non-injective matches, we have to modify a single point of the procedure. The definitions/outcomes presented in Section 7.3 remain valid. First, we deal with a possibly non-injective node map $m_{N}$, assuming the edge map $m_{E}$ injective. Then, we exemplify the treatment of a non-injective $m_{E}$, which, however, has little interest in the practice.

A non-injective match $m$ for the rule in Figure 5 identifies nodes 1,2 of $L$ in $G$. The reason why the rule's translation (Figure 6), in either form (a) or (b), excludes such a match, is that the corresponding instance $b_{m}$ of $t_{r}$ assigns

\footnotetext{
${ }^{10} C$ is a graph, because the gluing condition ensures that the source and target nodes of an edge in $C$ are nodes in $C$
} 
variables $n_{1}, n_{2} \in \operatorname{Var}_{I}$ the same color of $\mathrm{N}$ (denoting the image in $G$ of the two nodes of $L)$. In the original translation, the instance $\left(t_{r}, b_{m}\right)$ is not enabled because I [Node, $\left.t_{r}\right]\left(b_{m}\right)$ is a non-type-set bag; in the equivalent form, $\left(t_{r}^{\prime}, b_{m}\right)$ is not even a valid transition instance.

The workaround to consider injective matches, however, is unexpectedly simple. It consists of writing the function(s) I[Node, $\left.t_{r}\right]\left(\mathrm{O}\left[\right.\right.$ Node,$\left.\left.t_{r}\right]\right)$ as an elementary tuple with an inner summation, instead of a sum of elementary tuples, thus exploiting the "type-set" semantics of class-functions (Section 2.2.3).

Figure 9 shows an (injective) DPO rule with a non-injective match $m$ and the corresponding graph transformation. Figure 10 shows the rule's alternative translations into SN: (a) comes directly from Procedure 1, i.e., it refers to injective matches of the rule, (b) refers, instead, to matches $m$ with any $m_{N}$.

Passing from (a) to (b) requires two simple changes to color annotations: we erase variable $n_{3}\left(\operatorname{Var}_{I}^{*}=\left\{n_{3}\right\}\right)$ from I[Node, $\left.t_{r}\right], \mathrm{O}\left[\right.$ Node, $\left.t_{r}\right]$ then we rewrite the residual expression of the input (output) arc-function as:

$$
\langle n 1\rangle+\left\langle n_{2}\right\rangle F \stackrel{\not \equiv}{\rightarrow}\left\langle n_{1}+n_{2}\right\rangle
$$

Due to the class-function semantics, the resulting expression is type-set and evaluates as the original one (only) if $n_{1}, n_{2}$ are bound (map) to different colors. This way, we encode non-injective morphisms $L \rightarrow G$ as $t_{r}^{\prime}$ instances where two or more variables among $\left\{n_{1}, n_{2}, n_{3}\right\}$ are bound (map) to the same color.

As for the rule in Figure 5 we get its SN translation including matches $m$ with non-injective $m_{N}$ by just erasing the transition's guard from the SN in Figure 6 (b).

Based on the above remarks, to deal with rule matches $m$ with any $m_{N}$ we have to slightly modify Step 2 of Procedure 1 Note that revised Step 2 embeds the reduction on I/O arc-functions specified by Property 7 .

Procedure 1 (Procedure 1 with revised Step 2 - match $m$ with any $m_{N}$ )

$$
\mathrm{I}\left[\text { Node }, t_{r}\right]=\left\langle\sum_{n_{i} \in \operatorname{Var}_{I}^{-} \cup \operatorname{Var}_{\text {obs }}} n_{i}\right\rangle \quad \mathrm{O}\left[\text { Node }, t_{r}\right]=\left\langle\sum_{n_{i} \in \operatorname{Var}_{I}^{-} \cup \operatorname{Var}_{\text {fresh }}} n_{i}\right\rangle
$$

(H[Node, $\left.t_{r}\right]$ is unchanged)

Is is straightforward to find out the implicit predicates which characterize the enabled instances of the SN translation of a DPO rule $r$ including also non-injective matches of $r$.

Property 9 Let $t_{r}$ be the SN transition obtained by translating an injective DPO rule $r=L \stackrel{\phi_{L}}{\longleftarrow} I \stackrel{\phi_{R}}{\longrightarrow} R$ according to Procedure $1{ }^{*}$ and $\mathbf{m}$ a graph-encoding.

If $\left(t_{r}, b\right)$ is enabled in $\mathbf{m}$ then

$$
\forall n_{i} \in \operatorname{Var}\left(t_{r}\right) \forall n_{j} \in \operatorname{Var}\left(t_{r}\right)-\operatorname{Var}_{I}, i \neq j: n_{i}(b) \neq n_{j}(b)
$$




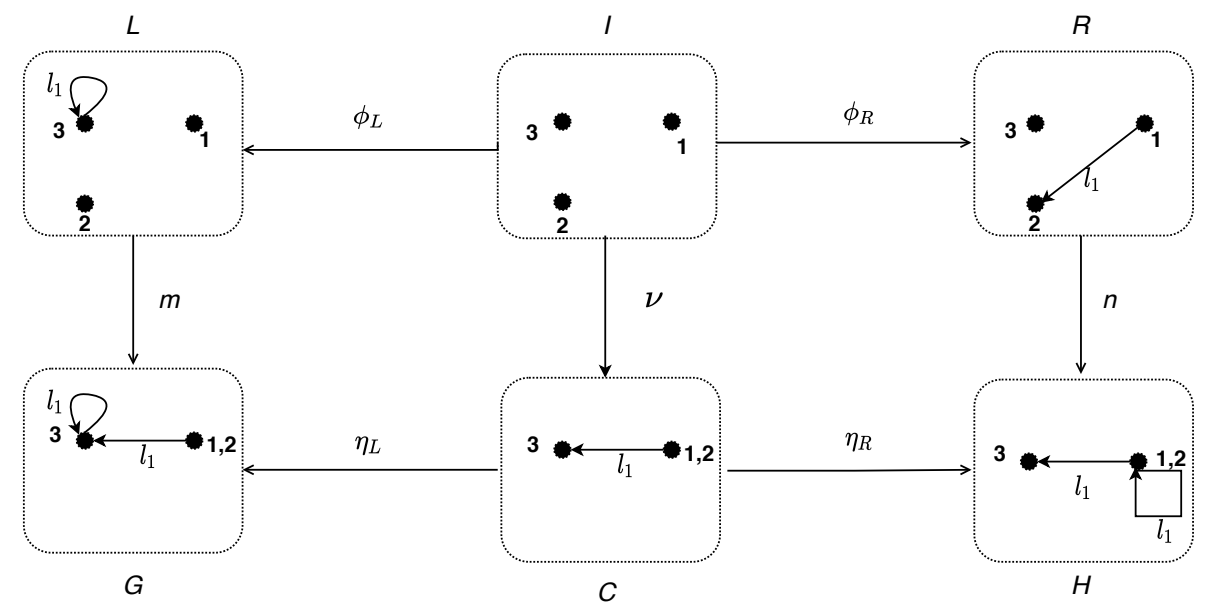

Figure 9: DPO rule with non-injective match $m_{N}$

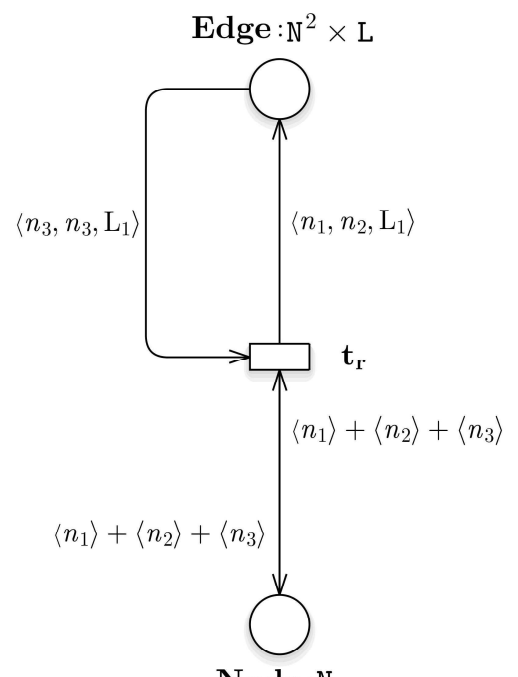

Node :N

(a)

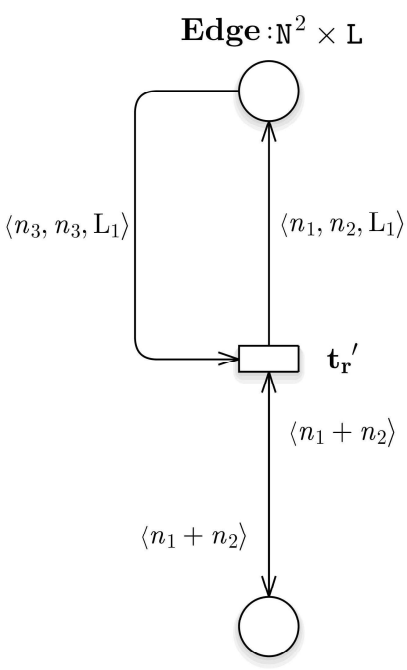

Node: $\mathrm{N}$

(b)

Figure 10: SN translations of rule in Figure 9 injective $m_{N}$ vs any $m_{N}$ 
A very important thing is that all the notions/outcomes presented in Section 7.2 still hold, except Property 6 (replaced by Property 9). This statement has two main explanations. As for the gluing condition, the few changes introduced to the translation procedure allow a valid instance of $t_{r}$ (corresponding to a non-injective match $m$ ) to identify only variables denoting non-obsolete nodes. As for the dangling-edge condition, the changes above do not even involve it. The second (related) reason lies in the new shape of the function I[Node, $\left.t_{r}\right]$ : this function is type-set, hence, also instances corresponding to non-injective morphisms may be enabled in a graph-encoding.

Non-injective $m_{E}$. Figure 11 shows a DPO rule whose associated match $m$ is such that $m_{E}$ is non-injective $\left(m_{E}\right.$ identifies the two edges with label $l b_{1}$ of $L$ in the host graph $G$ ). Figure 12 shows two alternative SN translations of the rule, one (a) obtained according to Procedure 1 (the functions on I/O arcs incident to place Node are null because $\operatorname{Var}_{I}^{-}=\emptyset$ ), the other (b) taking account of noninjective edge-matches $m_{E}$. The arc-function I[Edge, $t_{r}$ ] contains the sub-sum $F=\left\langle n_{1}, n_{3}, \mathrm{~L}_{1}\right\rangle+\left\langle n_{1}, n_{2}, \mathrm{~L}_{1}\right\rangle$, whose two terms represent edges of $L$ which may be identified by a morphism $m_{E}$, prior identification of their target nodes. Therefore, we rewrite the subsum into a (non-equivalent) disjoint form:

$$
F \stackrel{\not \equiv}{\rightarrow} F\left[n_{2} \neq n_{3}\right]+\left\langle n_{1}, n_{2}, \mathrm{~L}_{1}\right\rangle\left[n_{2}=n_{3}\right] \equiv\left\langle n_{1}, n_{2}+n_{3}, \mathrm{~L}_{1}\right\rangle
$$

whose elements represent injective/non-injective $m_{E}$, respectively. We can express this form compactly, utilizing the class-function $n_{2}+n_{3}$.

The general formalization is merely a technical matter: for each (maximal) subset of edges (symbolic tuples) $\left\{\left\langle n_{i}, n_{j}, \mathrm{~L}_{k}\right\rangle\right\}$ of graph $L$ with an identical labe ${ }^{11}$, we should consider all its partitions, and encode each of these (modelling any edge map) through a set of (in)equalities associated to a term of I[Edge, $t_{r}$ ] (we get mutually exclusive guards). Let us omit the details of this operation (combinatorial with respect to $\left|b a g_{E_{L}}\right|$ ), which is of little interest, both theoretical and practical. Even though non-injective edge-matches of a rule exhibit a kind of "folding" effect, they ultimately obscure rule's interpretation.

\subsection{Non-Injective DPO Rules}

Until now, we have assumed that both legs of a rule $r=L \stackrel{\phi_{L}}{\longleftarrow} I \stackrel{\phi_{R}}{\longrightarrow} R$ are injective. This is actually a general assumption. Finally, we consider the case of a non-injective right leg $\phi_{R}$, what may result in merging nodes/edges of a source graph $G$.

A non-injective rule and related graph transformation are shown in Figure 13 . The nodes 2 and 3 of the interface $I$, in fact, are identified by $\phi_{R}$, together with the two incident arcs. The rule's application to $G$, via the match $m$, results in $H=R+{ }_{I} C$ : elements of $R$ and $C$ with a common pre-image in $I$ are merged. As a result, nodes 2 and 3 of $G$ are merged, as well as two of the three edges

\footnotetext{
${ }^{11}$ identification of parallel edges of graph $L$ is coherently excluded
} 


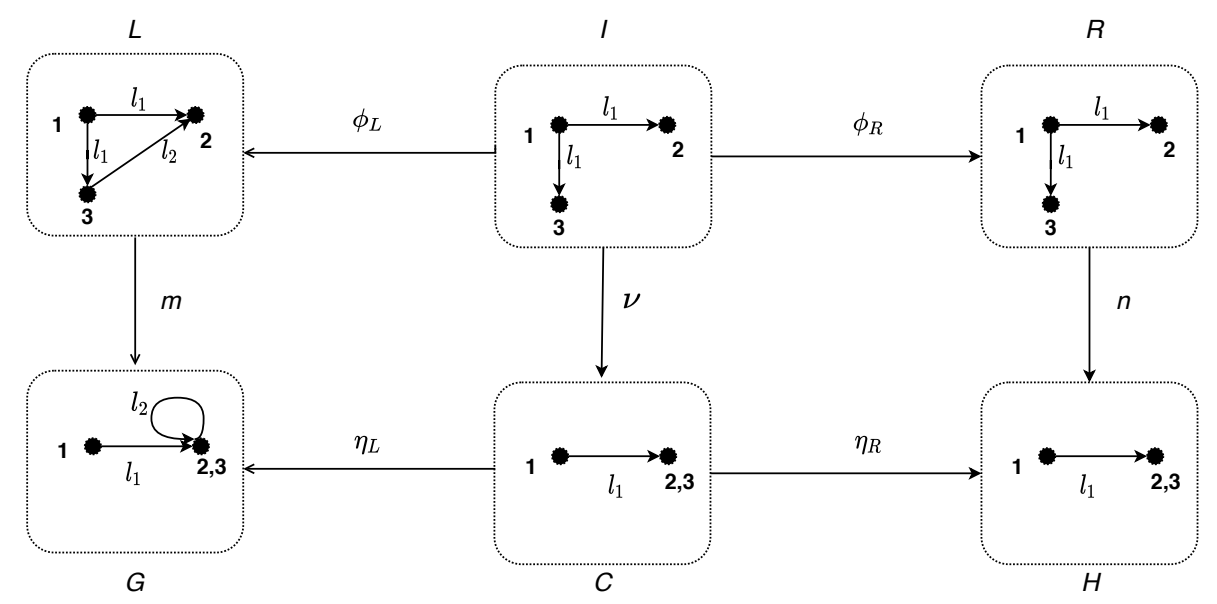

Figure 11: DPO rule with a non-injective match $m_{E}$ and graph transformation
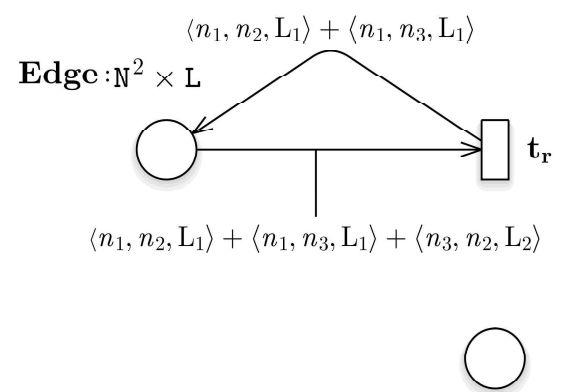

Node : N
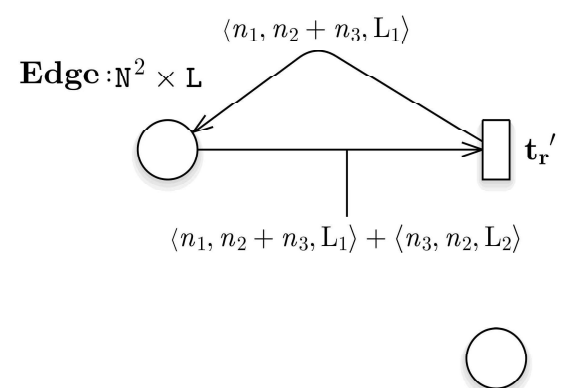

Node :N

(b)

Figure 12: SN translations of DPO rule in Figure 11 injective $m_{E}\left(\right.$ any $\left.m_{N}\right)$ vs any $m_{E}$ 


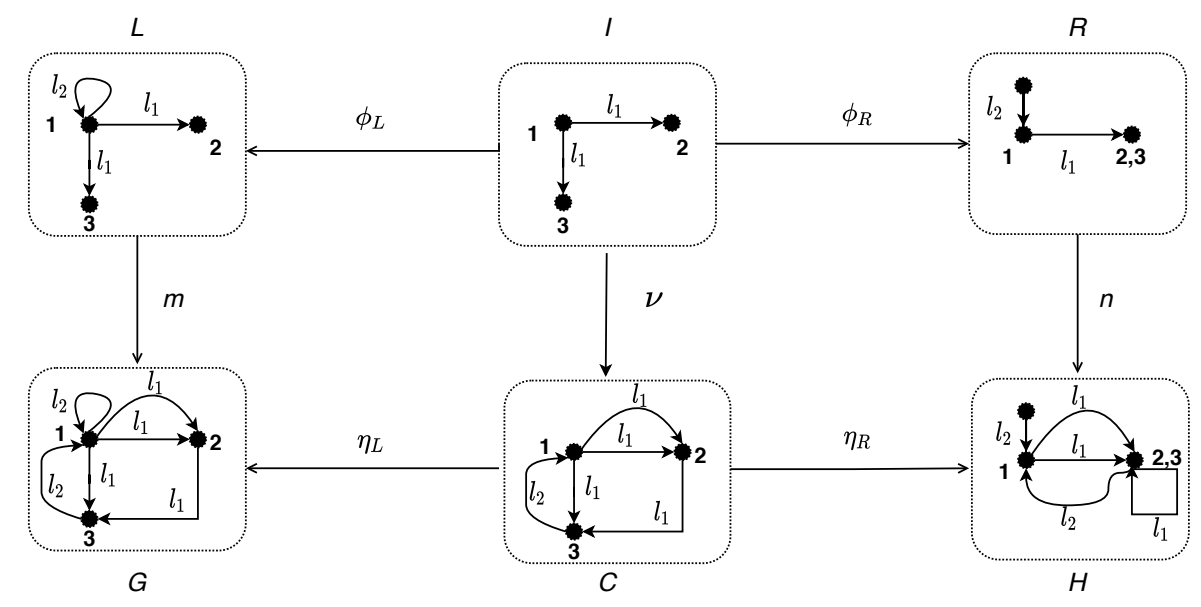

Figure 13: a NON-injective DPO rule and a graph transformation

(with label $l b_{1}$ ) departing from node 1 . The edges incident to nodes 2,3 of $G$ are "redirected" in $H$ to the node resulting from merge.

Being able to reproduce this context-dependent changes, preserving the atomic semantics of DPO rules, requires the use of a SN composite rule (Section 6). The subnet's observable transition, $t_{r}$, directly implements most of the structural changes on a source graph $G$ (merge/add/removal of elements), and triggers a sequence of immediate transitions which rebuild the adjacencies of merged nodes on the target graph $H$. The color annotations of $t_{r}$ are slightly different from the injective rule case, the rest of the subnet is built according to a structural pattern.

Figure 14 shows the SN subnet translating the rule in 13 . Let us formally describe the translation procedure, focusing on new elements.

Procedure 2 (SN translation of non-injective DPO rules - any match $m$ ) Let $r=L \stackrel{\phi_{L}}{\longleftarrow} I \stackrel{\phi_{R}}{\longrightarrow} R$ be a DPO rule, with $\phi_{R}$ non-injective and $\phi_{L}$ injective. We refer to any match $m$, with $m_{E}$ injective.

1. The rule's observable transition, $t_{r}$, is linked to places Node, Edge as described in Section 7.2, with a few differences:

- $\operatorname{Var}_{f r e s h} \cong\left(N_{R}-\phi_{R}\left(N_{I}\right)\right) \cup\left\{v \in N_{r},\left|\phi_{R}^{-1}(v)\right|>1\right\}$. In other words, the nodes of $R$ with non-singleton pre-images are described as fresh variables, denoted $V$ ar $r_{\text {merge }}$.

- I[Node, $\left.t_{r}\right]$, O[Node, $\left.t_{r}\right]$ are defined as in Step 2 of Procedure 1$]^{k}$.

- $\forall n_{i} \in \operatorname{Var}_{\text {merge }} \forall n_{j}, n_{k} \in \phi_{R}^{-1}\left(n_{i}\right), j \neq k$, there is $n_{j} \neq n_{k}$ in $g\left(t_{r}\right)$

(Note that in general $b a g_{E_{I}} \not \leq b a g_{E_{R}}$ )

2. for each $n_{i} \in \operatorname{Var}_{\text {merge }}$, there are a pair of places $\left\{\right.$ ToMerge $_{i}$, Merge $\left._{i}\right\}$ and a pair transitions $R_{i}=\left\{\right.$ reconnect_s $_{i}$, reconnect_t $\left.t_{i}\right\}$, such that, letting 
$x, y$, be auxiliary, type- $\mathrm{N}$ variables, and $t_{i}$ denote any $R_{i}$ element:

$$
\begin{aligned}
& \mathrm{O}\left[\text { ToMerge }_{i}, t_{r}\right]=\mathrm{I}\left[\text { ToMerge }_{i}, t_{i}\right]=\mathrm{O}\left[\text { ToMerge }_{i}, t_{i}\right]=\mathrm{I}\left[\text { ToMerge }_{i}, \text { end }\right]= \\
& \sum_{n_{j} \in \phi_{R}^{-1}\left(n_{i}\right)}\left\langle n_{j}\right\rangle \\
& \mathrm{O}\left[\text { Merge }_{i}, t_{r}\right]=\mathrm{I}\left[\text { Merge }_{i}, t_{i}\right]=\mathrm{O}\left[\text { Merge }_{i}, t_{i}\right]=\mathrm{I}\left[\text { Merge }_{i}, \text { end }\right]=\left\langle n_{i}\right\rangle \\
& \mathrm{I}\left[\text { Edge }, t_{i}\right]=\langle x, y, l\rangle \quad \mathrm{O}\left[\text { Edge, reconnect_s } \mathbf{s}_{i}\right]=\left\langle n_{i}, y, l\right\rangle \\
& \mathrm{O}\left[\text { Edge, } \text { reconnect_t }_{i}\right]=\left\langle x, n_{i}, l\right\rangle \\
& g\left(\text { reconnect_s }_{i}\right)=\bigvee_{n_{j} \in \phi_{R}^{-1}\left(n_{i}\right)} x=n_{j} \quad g\left(\text { reconnect_t }_{i}\right)=\bigvee_{n_{j} \in \phi_{R}^{-1}\left(n_{i}\right)} y=n_{j}
\end{aligned}
$$

3. transition end is linked to place Edge through an inhibitor arc

$$
\begin{aligned}
\mathrm{H}\left[\mathrm{Edge}_{i}, \text { end }\right] & =\langle F, \text { All }, \text { All }\rangle+\langle A l l-F, F, A l l\rangle \quad \text { where } \\
F & =\sum_{n_{i} \in \text { Var }_{\text {merge }}, n_{j} \in \phi_{R}^{-1}\left(n_{i}\right)}\left\langle n_{j}\right\rangle
\end{aligned}
$$

4. Places Start,Trigger are linked to the sub-net's transitions, as indicated at (the first two points of) Definition 4

The observable transition, $t_{r}$, manages the possible identification of edges by $\phi_{R}$, and the other rewritings involving obsolete/fresh graph elements (if any). The couple of transitions reconnect_s $s_{i}$, reconnect_t $t_{i}$ manage the redirection of edges incident to any of the (source/target) nodes which are merged into a fresh new one. As a last step, transition end removes all the merged nodes, according with the dangling-edge condition.

Since, by construction, all the transitions of the SN translating a noninjective DPO rule match Definition 2 (and the other conditions set by Definition 4, it holds:

Property 10 Let $r$ be a non-injective DPO rule. The SN subnet obtained from $r$ according to Procedure 2 is a SN composite GTR.

As for the example in Figure 13, we get: $\operatorname{Var}_{I}=\left\{n_{1}, n_{2}, n_{3}\right\}\left(\operatorname{Var}_{I}^{-}=\emptyset\right)$, $\operatorname{Var}_{\text {fresh }}=\left\{n_{4}, n_{5}\right\}, \operatorname{Var}_{\text {merge }}=\left\{n_{4}\right\}, \operatorname{Var}_{\text {obs }}=\emptyset, \phi_{R}^{-1}\left(n_{4}\right)=\left\{n_{2}, n_{3}\right\}$. The arc-functions and the guard annotating $t_{r}$ turn out to be:

$$
\begin{aligned}
& \mathrm{I}\left[\text { Node }, t_{r}\right]=\epsilon \quad \mathrm{O}\left[\text { Node }, t_{r}\right]=\mathrm{H}\left[\text { Node }, t_{r}\right]=\left\langle n_{4}+n_{5}\right\rangle \\
& \mathrm{I}\left[\text { Edge }, t_{r}\right]=\left\langle n_{1}, n_{1}, \mathrm{~L}_{2}\right\rangle+\left\langle n_{1}, n_{2}, \mathrm{~L}_{1}\right\rangle+\left\langle n_{1}, n_{3}, \mathrm{~L}_{1}\right\rangle \\
& \mathrm{O}\left[\text { Edge }, t_{r}\right]=\left\langle n_{5}, n_{1}, \mathrm{~L}_{2}\right\rangle+\left\langle n_{1}, n_{4}, \mathrm{~L}_{1}\right\rangle \quad \mathrm{H}\left[\text { Edge }, t_{r}\right]=\epsilon \\
& g\left(t_{r}\right)=n_{2} \neq n_{3} \wedge n_{4} \neq n_{5}
\end{aligned}
$$




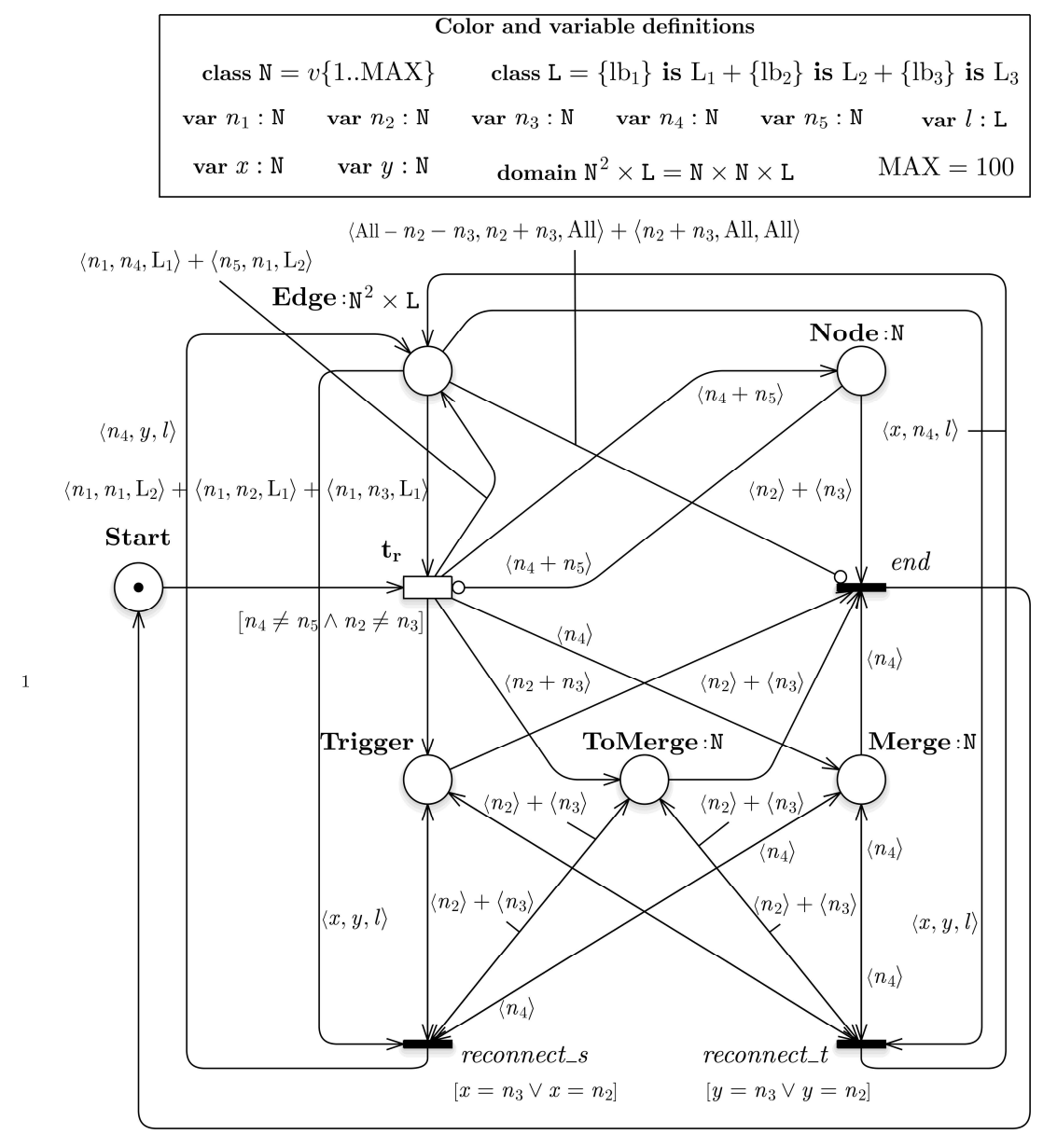

Figure 14: SN translation of rule in Figure 13 
The inequality $n_{2} \neq n_{3}$ in the guard of $t_{r}$ (Step 1 of Procedure 2 last point) makes the effect of a non-injective match $m$ not to overlap with that of the non-injective morphism $\phi_{R}$, which identifies nodes 2,3. Such an overlap would result in an undefined, or at least obscure, behaviour.

Accordingly, we need to modify (a bit) the match notion for non-injective DPO rules.

Definition 7 (match of a non-injective rule) Let $r=L \stackrel{\phi_{L}}{\longleftarrow} I \stackrel{\phi_{R}}{\longrightarrow} R$ be a rule with $\phi_{R}$ non-injective and $\phi_{L}$ injective, and $G$ be a graph.

A morphism $m: L \rightarrow G$ is a match if and only if $m_{E}$ is injective and

$\forall v_{i} \in N_{R},\left|\phi_{R}^{-1}\left(v_{i}\right)\right|>1 \forall v_{j}, v_{k} \in \phi_{L}\left(\phi_{R}^{-1}\left(v_{i}\right)\right), j \neq k: m_{N}\left(v_{j}\right) \neq m_{N}\left(v_{k}\right)$

We may thus easily extend to non-injective rules the notion of transition instance(s) corresponding to a match (Definition 6), by referring to the observable transition $t_{r}$ of the SN subnet translating one such rule.

We have to rephrase Corollary 1 by taking into account the fact that the observable transition triggers a sequence of immediate transitions.

Corollary 2 Let $r=L \stackrel{\phi_{L}}{\longleftarrow} I \stackrel{\phi_{R}}{\longrightarrow} R$ be a non-injective rule, $t_{r}$ the observable transition of the subnet obtained from $r$ according to Procedure 2, $G$ a graph.

If $m: L \rightarrow G$ satisfies the gluing condition and $G \stackrel{r, m}{\longrightarrow} H$ then $\mathbf{m}_{G}\left[t_{r}, b_{m} \cdot \rho\right\rangle \mathbf{m}_{G^{\prime}}$, where $\rho$ is a firing sequence of immediate transitions terminated by transition end and $H \cong G^{\prime}$. And vice versa.

A final note concerns the left leg $\phi_{L}$ of a rule. Almost all papers on graph transformation assume that it is injective. The reason is that, if $\phi_{L}$ is not injective, there may be cases when, even if a context $C$ does exist for a match $m$ of $L$ in a host graph $G$, it is not unique (for $m$ ). That may result in nondeterminism, because some nodes of $G$ may have to be split, and the incident edges may decide to "follow" any of split nodes. This situation shows very little practical interest. However, we conjecture it might be represented without any difficulty using composite rules, being in some sense dual to the case of a non-injective right leg $\phi_{R}$.

\subsection{From $S N$ GTR to DPO Rules}

We conclude the comparison between SN Graph Transformation Rules and DPO rules by shortly discussing the opposite direction, going from SN to DPO. In our opinion, it is much less intriguing. The main reason is that there are lots of rule application conditions, especially of restrictive type, that we can easily model in SN, instead we hardly, or even cannot, embed in a (base) DPO approach (or other algebraic ones.) The typical case consists of inhibiting conditions on the left-hand side of a rule, e.g., when computing graph transitive closure (whenever there exists an edge from $s$ to $v$ and one from $v$ to $t$, create one from $s$ to $t$ only if such an edge is absent). Other conditions we can easily set with SN (and cannot so easily with DPO) concern edge labels (e.g., removing 
edges with labels different from a given one), multiplicity (e.g, removing parallel instances of an edge, leaving a single one), and so forth.

We believe that it is possible to characterize a quite large subclass of SN GTR (especially of elementary type) that can be mechanically translated into corresponding DPO rules. This might be done by defining syntactical patterns for arc-functions that are likely matched by most practical examples: the SN rules shown in the gallery of Figure 1 (but R5), e.g., are easily translatable into DPO rules. This is part, however, of ongoing work.

\section{Conclusions and Ongoing Work}

In this paper, we have significantly enhanced and extended the outcome of a recent one [18] which, reversing the usual perspective, has proposed a formalization of Graph Transformation Systems (GTS) in terms of Symmetric Nets (SN). SN are a standard, High-level PN formalism featuring a compact syntax highlighting system behavioural symmetries, which are exploited in efficient analysis techniques. We use a structural approach, exploiting a recently implemented symbolic calculus for SN, to validate Graph Transformation Rules and formally verify properties of GTS in an efficient way. A major strength is given by the possibility of using automated tools for the editing and analysis of graph transformation models.

In the second part of the paper, we have provided a new semantic characterization of the SN-based graph transformation approach, by carrying out a thorough comparison with the classical, algebraic double-pushout (DPO) approach. We have shown in a constructive way that any DPO rule maps to a corresponding, elementary or composite, SN rule. We have treated both injective and non-injective DPO rules and rule matches. The comparison shows that the SN approach is, in some sense, a generalization of the DPO.

Throughout the paper, we have used simple but significant examples of graph encoding through SN. Edge-labelled, multi-graphs are considered.

Ongoing/Future Work. We are extending the SN-based graph transformation by encoding Place/Transitions $(\mathrm{P} / \mathrm{T})$ nets enriched with inhibitor arcs, a kind of directed, bipartite graphs. A SN emulator for $\mathrm{P} / \mathrm{T}$ nets (encoded as markings) has been defined in 30, together with a simple API for base net-transformations. We aim at integrating the SN emulator with the theoretically sound, rule-based graph transformation approach presented in this paper.

More general classes of graphs, in particular hypergraphs, cannot simply encoded with SN, due to the SN low data abstraction ability. On the other side, for the same reason, graph transformations with a richer (typed, constrained) labelling mechanism, generally known as Attributed Graph Rewriting [27, 28, are hardly translatable into SN. To cover more complex graph encodings and graph transformation approaches, Algebraic (Spec-inscribed) Petri nets 20] may be more conveniently used [31. Unfortunately, this formalism pays its higher expressivity with reduced analysis capability and automated support, so its usage requires further studies. 


\section{References}

[1] G. Rozenberg (Ed.), Handbook of Graph Grammars and Computing by Graph Transformation, WORLD SCIENTIFIC, 1997. doi:10.1142/3303

[2] H. Ehrig, G. Engels, H.-J. Kreowski, G. Rozenberg (Eds.), Handbook of Graph Grammars and Computing by Graph Transformation: Vol. 2: Applications, Languages, and Tools, WORLD SCIENTIFIC, 1999. doi:10.1142/4180.

[3] H. Ehrig, M. Pfender, H. J. Schneider, Graph-Grammars: An Algebraic Approach, in: Proceedings of the 14th Annual Symposium on Switching and Automata Theory (Swat 1973), SWAT '73, IEEE Computer Society, USA, 1973, p. 167-180. doi:10.1109/SWAT.1973.11

[4] A. Corradini, U. Montanari, F. Rossi, H. Ehrig, R. Heckel, M. Löwe, Algebraic approaches to graph transformation - part i: Basic concepts and double pushout approach, in: Rozenberg 1, pp. 163-245. doi: $10.1142 / 9789812384720 \_0003$.

[5] H. Ehrig, K. Ehrig, U. Prange, G. Taentzer, Fundamentals of algebraic graph transformation., Berlin: Springer, 2006. doi:10.1007/ 3-540-31188-2

[6] V. Danos, J. Feret, W. Fontana, R. Harmer, J. Hayman, J. Krivine, C. Thompson-Walsh, G. Winskel, Graphs, Rewriting and Pathway Reconstruction for Rule-Based Models, in: S. D. L.-Z. fuer Informatik (Ed.), FSTTCS 2012 - IARCS Annual Conference on Foundations of Software Technology and Theoretical Computer Science, Vol. 18 of LIPIcs, Hyderabad, India, 2012, pp. 276-288. doi:10.4230/LIPIcs.FSTTCS.2012.276.

[7] M. Fernández, H. Kirchner, B. Pinaud, J. Vallet, Labelled graph rewriting meets social networks, in: D. Lucanu (Ed.), Rewriting Logic and Its Applications, Springer International Publishing, Cham, 2016, pp. 1-25. doi:10.1007/978-3-319-44802-2_1.

[8] K. Do, T. Tran, S. Venkatesh, Graph transformation policy network for chemical reaction prediction, in: Proceedings of the 25th ACM SIGKDD International Conference on Knowledge Discovery \& Data Mining, KDD '19, Association for Computing Machinery, New York, NY, USA, 2019, p. 750-760. doi:10.1145/3292500.3330958.

[9] W. Reisig, Petri Nets: An Introduction, Springer-Verlag New York, Inc., New York, NY, USA, 1985. doi:10.1007/978-3-642-69968-9.

[10] W. Fokkink, Introduction to process algebra., Berlin: Springer, 2000.

[11] D. Sangiorgi, D. Walker, The $\pi$-calculus: A theory of mobile processes., Cambridge: Cambridge University Press, 2001. 
[12] H. Ehrig, B. König, Deriving bisimulation congruences in the DPO approach to graph rewriting with borrowed contexts., Math. Struct. Comput. Sci. 16 (6) (2006) 1133-1163.

[13] J. Padberg, L. Kahloul, Overview of Reconfigurable Petri Nets, in: R. Heckel, G. Taentzer (Eds.), Graph Transformation, Specifications, and Nets: In Memory of Hartmut Ehrig, Springer International Publishing, Cham, 2018, pp. 201-222. doi:10.1007/978-3-319-75396-6_11.

[14] H.-J. Kreowski, A comparison between Petri-nets and graph grammars, in: H. Noltemeier (Ed.), Graphtheoretic Concepts in Computer Science, Springer Berlin Heidelberg, Berlin, Heidelberg, 1981, pp. 306-317.

[15] A. Corradini, Concurrent graph and term graph rewriting, in: U. Montanari, V. Sassone (Eds.), CONCUR '96: Concurrency Theory, Springer Berlin Heidelberg, Berlin, Heidelberg, 1996, pp. 438-464. doi:10.1007/ 3-540-61604-7_69.

[16] P. Baldan, A. Corradini, F. Gadducci, U. Montanari, From Petri Nets to Graph Transformation Systems, ECEASST 26 (01 2010). doi:10.14279/ tuj.eceasst.26.368.

[17] H. Ehrig, J. Padberg, Graph Grammars and Petri Net Transformations, in: J. Desel, W. Reisig, G. Rozenberg (Eds.), Lectures on Concurrency and Petri Nets: Advances in Petri Nets, Springer Berlin Heidelberg, Berlin, Heidelberg, 2004, pp. 496-536. doi:10.1007/978-3-540-27755-2_14.

[18] L. Capra, An operational semantics of graph transformation systems using symmetric nets, in: M. Marin, A. Crăciun (Eds.), Proceedings Third Symposium on Working Formal Methods, Timişoara, Romania, 3-5 September 2019, Vol. 303 of Electronic Proceedings in Theoretical Computer Science, Open Publishing Association, 2019, pp. 107-119. doi:10.4204/EPTCS. 303.8

[19] G. Chiola, C. Dutheillet, G. Franceschinis, S. Haddad, Stochastic wellformed colored nets and symmetric modeling applications, IEEE Transactions on Computers 42 (11) (1993) 1343-1360. doi:10.1109/12.247838.

[20] K. Jensen, G. Rozenberg (Eds.), High-level Petri Nets: Theory and Application, Springer-Verlag, London, UK, 1991. doi:10.1007/ 978-3-642-84524-6.

[21] K. Jensen, Coloured Petri Nets. Basic Concepts, Analysis Methods and Practical Use., Volume 1, Basic Concepts. Monographs in Theoretical Computer Science, Springer-Verlag, 2nd corrected printing 1997. ISBN: 3-54060943-1., 1997. doi:10.1007/978-3-662-03241-1.

[22] E. G. Amparore, G. Balbo, M. Beccuti, S. Donatelli, G. Franceschinis, 30 years of GreatSPN, in: Principles of Performance and Reliability Modeling and Evaluation, Springer, 2016, pp. 227-254. doi:10.1007/ 978-3-319-30599-8_9. 
[23] G. Chiola, C. Dutheillet, G. Franceschinis, S. Haddad, A symbolic reachability graph for coloured Petri nets, Theoretical Computer Science 176 (1) (1997) 39 - 65. doi:10.1016/S0304-3975(96)00010-2.

[24] L. Capra, M. D. Pierro, G. Franceschinis, A High Level Language for Structural Relations in Well-Formed Nets, in: Proc. of the 26th Int. Conf. ATPN 2005, Vol. LNCS 3536, Springer, 2005, pp. 168-187. doi: 10.1007/11494744_11.

[25] L. Capra, M. De Pierro, G. Franceschinis, Computing Structural Properties of Symmetric Nets, in: J. Campos, B. R. Haverkort (Eds.), Quantitative Evaluation of Systems QEST 2015, Springer International Publishing, Cham, 2015, pp. 125-140. doi:10.1007/978-3-319-22264-6_9.

[26] L. Capra, M. De Pierro, G. Franceschinis, SNexpression: A Symbolic Calculator for Symmetric Net Expressions, in: R. Janicki, N. Sidorova, T. Chatain (Eds.), Application and Theory of Petri Nets and Concurrency, Springer International Publishing, Cham, 2020, pp. 381-391. doi: 10.1007/978-3-030-51831-8_19.

[27] B. König, V. Kozioura, Towards the verification of attributed graph transformation systems, in: H. Ehrig, R. Heckel, G. Rozenberg, G. Taentzer (Eds.), Graph Transformations, Springer Berlin Heidelberg, Berlin, Heidelberg, 2008, pp. 305-320. doi:10.1007/978-3-540-87405-8_21.

[28] F. Orejas, Symbolic graphs for attributed graph constraints., J. Symb. Comput. 46 (3) (2011) 294-315.

[29] M. Löwe, Algebraic approach to single-pushout graph transformation., Theor. Comput. Sci. 109 (1-2) (1993) 181-224.

[30] L. Capra, M. Camilli, Towards Evolving Petri Nets: a Symmetric Netsbased Framework, IFAC-PapersOnLine 51 (7) (2018) 480 - 485, 14th IFAC Workshop on Discrete Event Systems WODES 2018. doi:10.1016/j. ifacol.2018.06.343.

[31] L. Capra, A pure SPEC-inscribed PN model for reconfigurable systems, in: 2016 13th International Workshop on Discrete Event Systems (WODES), IEEE, 2016, pp. 459-465. doi:10.1109/WODES.2016.7497888.

[32] L. Capra, M. D. Pierro, G. Franceschinis, A Tool for Symbolic Manipulation of Arc Functions in Symmetric Net Models, in: Proceedings of the 7th International Conference on Performance Evaluation Methodologies and Tools, ValueTools '13, ICST, Torino, Italy, 2013, pp. 320-323. doi:10. 4108/icst.valuetools.2013.254407.

[33] C. Dutheillet, S. Haddad, Conflict Sets in Colored Petri Nets, in: proc. of Petri Nets and Performance Models, 1993, pp. 76-85. doi:10.1109/PNPM. 1993.393433. 